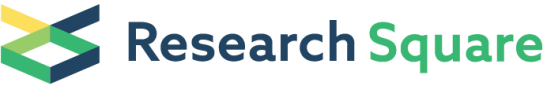 \\ Preprints are preliminary reports that have not undergone peer review. \\ They should not be considered conclusive, used to inform clinical practice, or referenced by the media as validated information.
}

\section{Oncoprotein CIP2A controls PKM2 dimer-tetramer transition through phosphorylation of serine 287 in non-small cell lung cancer}

\section{Li-Jun Liang}

Chinese Academy of Medical Sciences Cancer Institute and Hospital

\section{Yan-Fei Zhang}

Institute of Zoology, Chinese Academy of Sciences

\section{Di Wang}

Chinese Academy of Medical Sciences Cancer Institute and Hospital

\section{Fu-Ying Wang}

Chinese Academy of Medical Sciences Cancer Institute and Hospital

\section{Da-Wei Xie}

Chinese Academy of Medical Sciences Cancer Institute and Hospital

\section{Hong Yu}

Institute of Zoology, Chinese Academy of Sciences

\section{Zheng Wang}

Chinese Academy of Medical Sciences Cancer Institute and Hospital

\section{Bei-Bei Sun}

Chinese Academy of Medical Sciences Cancer Institute and Hospital

\section{San-Hui Gao}

Chinese Academy of Medical Sciences Cancer Institute and Hospital Hua Guo

Chinese Academy of Medical Sciences Cancer Institute and Hospital

\section{Xin-Chao Zhao}

Institute of Zoology, Chinese Academy of Sciences

\section{Gui-Zhen Wang}

Chinese Academy of Medical Sciences Cancer Institute and Hospital

\section{Guang-Biao Zhou ( $\sim$ gbzhou@cicams.ac.cn )}

Chinese Academy of Medical Sciences Cancer Institute and Hospital https://orcid.org/0000-00026327-2316

\section{Article}

Keywords: CIP2A, PKM2 dimer-tetramer transition, phosphorylation, glycolysis, non-small cell lung cancer 
Posted Date: January 19th, 2022

DOI: https://doi.org/10.21203/rs.3.rs-1232624/v1

License: (c) (i) This work is licensed under a Creative Commons Attribution 4.0 International License. Read Full License 


\section{Abstract}

Dimer-tetramer transition determines the pyruvate kinase activity of pyruvate kinase M2 (PKM2), the final rate-limiting enzyme in glycolysis. Several small molecules have been shown to be able to promote PKM2 confirmation change, but the role of oncoproteins including the cancerous inhibitor of protein phosphatase PP2A (CIP2A) that is overexpressed in most human malignancies, in PKM2 dimer-tetramer transition remains largely unknown. Here we reported that CIP2A inhibited glycolysis and promoted oxidative metabolism in non-small cell lung cancer (NSCLC) cells. CIP2A directly bound PKM2 and induced formation of PKM2 tetramer, whereas CIP2A depletion resulted in reduced tetrameric and increased dimeric forms of PKM2. We found that serine 287 of PKM2 was essential for PKM2 dimertetramer switching, whereas mutation in this residue abrogated CIP2A-induced tetramer formation and pyruvate kinase activity increment. Clinically, the expression of CIP2A was positively associated with the level of phosphorylated PKM2 S287, and CIP2 $A^{\text {high }} / P K M 2^{\text {high }}$ and $P K M 2^{\text {high }} / C I P 2 A^{\text {high }}$ were associated with poor prognosis of NSCLC patients. Pharmacological inhibition of glycolysis or activation of PKM2 synergistically inhibited NSCLC cell growth when combined with CIP2A-targeting compound celastrol both in vitro and in vivo. These results demonstrated that CIP2A determines PKM2 dimer-tetramer transition and controls the metabolic reprogramming of NSCLC cells.

\section{Introduction}

Lung cancer is the leading cause of cancer-related deaths worldwide, which accounts for $18 \%$ (approximately 1.8 million) of all cancer deaths in $2020^{1}$, with non-small-cell lung cancer (NSCLC) representing the major histologic subtype and accounting for $85 \%$ of all lung cancer cases. In recent decades, small molecular inhibitors based on oncogenic driver mutations or fusion status [i.e., epidermal growth factor receptor (EGFR) and anaplastic lymphoma kinase (ALK)] have increased the life expectancy and changed the treatment paradigm of NSCLCs. However, acquired drug resistance accompanied with undruggable mutations is always inevitable ${ }^{2}$. Although immune checkpoint blockades (ICBs), such as inhibitors of programmed cell death protein 1 (PD-1) and programmed deathligand 1 (PD-L1), have improved outcomes among patients without EGFR or ALK mutations, only approximately $20 \%$ of patients achieve a durable response $3,4,5$. Currently, the 5 -year survival rate for newly diagnosed NSCLC patients is still only $18.6 \%^{1,6}$. Therefore, novel therapeutic approaches or combination therapies for NSCLC are urgently needed.

Cancerous inhibitor of protein phosphatase 2A (CIP2A), an autoantigen previously known as KIAA1524 or p90 ${ }^{7}$, inhibits the catalytic phosphatase activity of the protein phosphatase 2A (PP2A) complex by competing with its substrate in binding to the holoenzyme ${ }^{8}$. CIP2A is overexpressed in over $70 \%$ of solid or hematological malignancies, including NSCLC, and is associated with high-grade cancer and poor prognoses of the patients ${ }^{9}$. CIP2A has the potential to be a useful biomarker for predicting treatment response and clinical outcomes after chemotherapy or targeted therapeutics ${ }^{10,11}$. Mechanismly, CIP2A functions as a prerequisite for the transformation of normal cells into tumor cells ${ }^{8}$, and CIP2A deficiency 
in mice leads to resistant to DMBA-induced basal-like breast cancer formation ${ }^{12}$. CIP2A enhances oncogenic activity of Myc and E2F1 and forms an oncogenic CIP2A-feedforward loop to promote malignant transformation and progression $8,13,14$. Enhanced expression of CIP2A/MycN drives neural crest toward a neural stem cell-like fate and facilitates priming of neuroblastoma ${ }^{15}$. CIP2A modulates cell-cycle progression ${ }^{16,17}$, whereas inhibition of CIP2A induces cell cycle arrest, promotes apoptosis or cellular senescence, and exhibits therapeutic efficacies in vitro and in vivo ${ }^{18,19}$. However, the role of CIP2A in cancer metabolism remains largely unknown.

Pyruvate kinase M (PKM) is the final rate-limiting enzyme in glycolysis, a hallmark of cancer ${ }^{20}$, which catalyzes the conversion of phosphoenolpyruvate (PEP) to pyruvate with concomitant formation of ATP. The PKM gene encodes two alternative-splicing products, PKM1 and PKM2, by the mutually exclusive use of exons 9 and $10^{21}$. PKM2 structurally exists as monomer, dimer, or tetramer. The tetrameric PKM2 exerts pyruvate kinase (PK) catalytic activity, whereas the nuclear dimeric PKM2 serves as a transcriptional coactivator or protein kinase to promote aerobic glycolysis and tumor progression $22,23,24$. PKM2 is conformationally coordinated by endogenous metabolic intermediates or cofactor bindinginduced posttranslational modification $25,26,27,28,29,30$. For example, the acetyltransferase p300 directly binds to PKM2 and acetylates PKM2 at K433, causing PKM2 to dissociate into dimers by interfering with the binding to its allosteric activator fructose 1,6-bisphosphate (FBP) ${ }^{31}$. Some modification sites (for example, S37 phosphorylated by EGFR) are located far away from the FBP binding pocket or dimerdimer interface, can also impact PKM2 oligomerization with unclear mechanism ${ }^{32}$. In contrast, serine can bind to PKM2 and facilitate tetramer formation to activate this enzyme, whereas serine deprivation leads to reduced PKM2 activity in cells ${ }^{29}$. A small molecule succinylaminoimidazolecarboxamide ribose5 -phosphate (SAICAR) induces PKM2 dimer-tetramer switching and enhances its pyruvate kinase activity

${ }^{30}$. However, cancer is addicted to gain-of-function oncoprotein and loss-of-function tumor suppressor signal pathways, but the roles of oncoproteins and tumor suppressors in PKM2 dimer-tetramer switching remain largely unknown.

In this study, we investigated the potential proteins that interact with CIP2A and found that CIP2A binds and phosphorylates PKM2 at S287, leading to the stabilization of tetramers and an increase in the pyruvate kinase activity of PKM2 in NSCLC, with reduced nuclear PKM2 and aerobic glycolysis. Our results revealed the novel functions of CIP2A in the regulation of key metabolic enzyme and its contribution to the Warburg effect in tumor cells and provided potential therapeutic implications for NSCLC treatment.

\section{Results}

\section{CIP2A deficiency activates glycolytic metabolism in NSCLC cells}

Our previous work showed that CIP2A was elevated and had an important role in human NSCLC 19, 33, 34 . During daily culture of NSCLC cell lines, we observed that the medium of CIP2A-knockdown cells turned 
orange much more rapidly than that of control cells, even at the same confluence (Fig. 1a). This observation indicated an increased production of acidic metabolites in CIP2A-knockdown cells, which prompted us to investigate the role of CIP2A in glucose metabolism. We applied XFe Seahorse energy metabolic stress assays to determine the impact of CIP2A on glucose metabolism, and the rate of glycolysis was assessed by ECAR. As anticipated, the glycolysis level, maximum glycolytic capacity, and glycolytic reserve were significantly increased in CIP2A-knockdown H1299 cells (Fig. 1b), whereas the reverse was observed in CIP2A-overexpressing cells (Fig. 1C). In CIP2A knockdown H1299 cells, a significant reduction in the basal mitochondrial respiration level, maximum rate of oxidative phosphorylation (OXPHOS) and reserve capacity of OXPHOS, as measured by OCR, were observed (Fig. 1d). In contract, a significant increase in the basal mitochondrial respiration level, maximum rate of OXPHOS and reserve capacity of OXPHOS, were seen in $\mathrm{H} 1299$ cells that were transfected with $\mathrm{PCDH}$ CMV-CIP2A (Fig. 1e).

\section{Identification of the glycolytic enzyme PKM2 as an endogenous CIP2A-associated protein}

To gain mechanistic insights, endogenous CIP2A was immunoprecipitated from extracts of A549 and $\mathrm{H} 1299$ cells, the eluates were resolved by SDS/PAGE, and several major bands not seen in the control lane were silver-stained and subjected to MS sequencing to identify CIP2A-interacting proteins. Interestingly, PKM was discovered as a CIP2A-binding protein (Fig. 2a). To verify this interaction, coimmunoprecipitation (Co-IP) analyses were performed using cell lysates of A549 and H1299 cells, and we showed that CIP2A could pull down PKM2 and vice versa (Fig. 2b). Additionally, GST-CIP2A could pull down PKM2 from H1299 cell lysates (Fig. 2C), suggesting that CIP2A could interact directly with PKM2. We detected whether CIP2A interacts with PKM1, despite the much lower expression of PKM1 in NSCLC cells than in SCLC cells (Fig. 2d), which was consistent with a previous study ${ }^{35}$. We found that PKM1 was unable to interact with CIP2A (Fig. 2e). To further confirm the interaction between CIP2A and PKM2, we detected the colocalization of CIP2A and PKM2 by IF staining in A549 and $\mathrm{H} 1299$ cells. The results indicated that CIP2A and PKM2 were primarily localized in the cytoplasm and that CIP2A colocalized with PKM2 (Fig. 2f). We next determined the interacting domain for the CIP2A-PKM2 interaction. As shown in Fig. $2 \mathrm{~g}$, a series of truncated mutants fused to the HA tag for PKM2 were generated to test the binding affinity with Flag-tagged CIP2A in 293T cells by Co-IP. We showed that deletion of the A2 domain (amino acids between 218 and 388) abrogated the interaction between PKM2 and CIP2A, whereas the other fragments of PKM2 retained the ability to bind CIP2A with various affinities (Fig. $2 \mathrm{~h}$ ). These results demonstrated that CIP2A could bind PKM2 at the A2 domain, and the functional consequence of this interaction warrants further investigation.

\section{CIP2A stabilizes PKM2 oligomerization}

To explore how CIP2A regulates PKM2, we first found that knocking down CIP2A had no effect on the total PKM2 protein expression (Fig. S1a). Then, we clarified whether CIP2A influences PKM2 oligomerization and pyruvate kinase activity. Size exclusion chromatography clearly indicated that the tetrameric formation of PKM2 was blocked in the shCIP2A group compared with the control group in 
A549 cells (Fig. 3a), and CIP2A overexpression skewed PKM2 towards high-molecular-weight forms in 293T cells (Fig. 3b). Further crosslinking experiments revealed that PKM2 migrated as a single band at $\sim 60 \mathrm{kDa}$ (monomer) in the absence of crosslinking reagent, indicating that SDS treatment resulted in a complete dissociation of potential PKM2 multimers (Fig. 3c). Following crosslinking conditions $(0.01 \%$ glutaraldehyde at $37^{\circ} \mathrm{C}$ for 9 minutes), tetrameric (240 kDa) PKM2 was increased after CIP2A overexpression in $\mathrm{H} 1299$ cells (Fig. 3c). Furthermore, the tetramer was significantly reduced and replaced by dimeric ( $120 \mathrm{kDa}$ ) and monomeric forms after CIP2A knockdown in H1299 cells (Fig. 3d). To validate whether CIP2A influences the interaction between the subunits of PKM2 in cells, we found that the amount of endogenous PKM2 that coprecipitated with HA-PKM2 was reduced in cells transfected with shCIP2A (Fig. 3e). These observations suggested that CIP2A facilitates PKM2 tetramer assembly.

\section{CIP2A enhances pyruvate kinase activity and hinders the nuclear translocation of PKM2}

We then assessed whether the PK activity of PKM2 is interfered with CIP2A. In vitro reactions showed that purified CIP2A protein from bacteria increased bacterial PK activity of PKM2 in a dose-dependent manner (Fig. 3f). On the contrary, there was a nearly $50 \%$ decrease in PKM2 activities in lysates of CIP2A-depleted $\mathrm{H} 1299$ and A549 cells (Fig. 3g). In addition, PK activity was decreased in H1299 cells treated with the PKM-specific inhibitor Compound $3 \mathrm{~K}^{36}$, and this response was attenuated by CIP2A overexpression (Fig. S1b). To further validate the cellular distribution of PKM2, we analyzed confocal microscopy images by comparing the PKM2 signaling intensity overlapping with high DAPI staining (nuclear region) by ZEN software ${ }^{37}$. A higher-intensity PKM2 signal from the nucleus was visualized in $\mathrm{H} 1299$ cells after knockdown of CIP2A (Fig. 3h). Consistent with the IF findings, western blot analysis of subfractionated cellular compartments indicated that CIP2A deficiency substantially induced the nuclear accumulation of PKM2 in the lysates collected from H1299 and A549 cells (Fig. 3i). The mild reduction of PKM2 in the cytosolic fractions under CIP2A deletion may be responsible for the nuclear PKM2 is indeed a small portion compared with the quantity of cytoplasmic PKM2 (Fig. 3i). These results indicated that CIP2A increases PK activity and inhibits the nuclear translocation of PKM2.

Metabolic flux analysis was further carried out to characterize the involvement of PKM2 in metabolic reprogramming regulated by CIP2A. The PKM2-specific activator TEPP- $46^{38}$ entirely restored the PK activity reduced by CIP2A knockdown (Fig. 3j) and abolished the sh CIP2A-mediated ECAR elevation (Fig. 3k). Consistent with the altered glycolysis phenotype, CIP2A deficiency-inducible mitochondrial respiration reduction, as measured by OCR, was blocked by TEPP-46 treatment (Fig. S1c). Additionally, TEPP-46 also reversed the acidic culture medium induced by CIP2A depletion in A549 cells (Fig S1d). These data suggested that CIP2A increases PK activity and impedes the nuclear translocation of PKM2, which supports the notion that CIP2A modulates metabolic reprogramming in NSCLC cells by intervening in multiple aspects of the PKM2 state and functions.

\section{PP2A regulatory subunit B56a controls PKM2 activity and reprogrammed glycolysis}


PP2A is a ubiquitously expressed heterotrimer containing the scaffolding A subunit, the catalytic $C$ subunit, and the substrate specificity-determining regulatory $B$ subunit ${ }^{39}$, and CIP2A inhibits the phosphatase activity of $P P 2 A^{8}$. We found that SiCIP2A resulted in reduced tetrameric and increased dimeric/monomeric PKM2, whereas knockdown of PP2A A subunit by siRNA increased tetrameric and reduced dimeric/monomeric PKM2, and co-transfection of siPP2A-Aa/ $\beta$ attenuated the effects induced by CIP2A depletion (Fig. S2a). The B' family of PP2A regulatory subunits is composed of a large array of different members, and only a subset of the PP2A subunit directly interacts with and is inhibited by CIP2A ${ }^{40}$. We validated which $B$ subunit mediates the modulation of PKM2 activity by CIP2A. We identified that only B56a (PPP2R5A) interacts with ectopic expression of HA-PKM2 (Fig. 4a). Co-IP analysis of endogenous proteins indicated that B56a, but not B56Y (PPP2R5C) or B56ع (PPP2R5E), interacted with PKM2 (Fig. 4b). Further IF analysis demonstrated colocalization between B56a and PKM2 in A549 and H1299 cells (Fig. 4c). These results suggested that CIP2A, PKM2 and PP2A might form a ternary complex (Fig. 4d) to regulate cell metabolism.

A short linear motif, [LMFI]xx[ILV]xEx (where $\mathrm{x}$ is any amino acid), acts as the preferred docking site for the complementary pocket conserved in the B56 subunit, whereas substitution of the two best

consensus residues at positions 1 and 4 to alanine would remarkably reduce the binding affinity ${ }^{41,42}$. We therefore analyzed the amino acid sequence of PKM2 and found two possible B56a binding motifs in the A2 domain (Fig. 4e). We constructed two mutated versions of the two potential binding motifs and found that F280A/I283A, but not L218A/V221A, completely disrupted the interaction between B56a and HA-PKM2, which could be the binding site (Fig. 4f).

We tested the effects of B56a on PKM2 PK activity and found that depletion of B56a notably upregulated the PKM2 PK activity reduced by CIP2A knockdown (Fig. 4g). Importantly, B56a deficiency substantially blocked CIP2A depletion-elicited PKM2 nuclear distribution and ECAR (Fig. 4h, i). In parallel, the increased OXPHOS level (Fig. S2b) and the yellow discoloration of the culture medium (Fig. S2c) due to CIP2A depletion were also reversed by co-depletion of B56a. These results indicate that the regulation of multiple functions of PKM2 and metabolic reprogramming are dependent on PP2A and specifically on the B56a regulatory subunit.

\section{PKM2 serine 287 is a novel phosphorylation site}

We next assessed the potential B56a-mediated posttranslational modifications of PKM2. Given that many known phosphorylation sites were found in the vicinity of the consensus sequence, especially the $\mathrm{S} / \mathrm{T}$ residues at positions 2/7/8/9, we generated Ala substitution mutants of Ser harbored near the two motifs and transfected into 293T cells with these mutants individually. The mutated proteins were purified by IP followed by western blotting with an anti-phosphoserine antibody. We found that mutation of S287 significantly reduced PKM2 phosphorylation, while the other 3 mutants only slightly reduced the overall phosphorylation of PKM2 (Fig. 5a). Additionally, MS also suggested that S287 is the most promising candidate serine phosphorylation site (Fig. 5b), and a genomic analysis showed that S287 is 
highly conserved among different species through evolution (Fig. 5c), demonstrating that S287 is a major phosphorylation site under this condition.

To study whether the phosphorylation status at S287 affects CIP2A/PP2A-mediated dimer/tetramer conversion of PKM2, we fractionated 293T cell lysates by gel filtration analysis and found that the distribution of total WT PKM2 spread throughout multiple fractions and that coexpression of CIP2A further switched PKM2 from monomer and dimer to tetramer (Fig. 5d). Notably, S287-phosphorylated PKM2 was only detected in a low molecular-weight fraction, and ectopic expression of CIP2A failed to shift fractions of PKM2 to higher molecular weight complexes (Fig. 5d). These results demonstrated a strong effect of phosphorylation at S287 in promoting tetramerization of PKM2.

\section{CIP2A modulates PKM2 phosphorylation at S287}

To study whether S287 is phosphorylated in vivo, we generated an antibody specific to phospho-S287 and carried out a series of experiments to test its specificity. The dot plot assay showed that the PKM2 phospho-S287 antibody preferentially detected the phosphorylated peptide but not the unmodified peptide and the signal gradually improved with increasing peptide concentration (Fig. S3a). Western blotting and $\mathrm{IHC}$ assays indicated that the staining was absent when the antibody was preincubated and neutralized with the phosphorylated peptide compared with the unmodified peptide (Fig. S3b, c). In parallel, when PKM2 was knocked down by expression of a shRNA, the band disappeared, as detected by the phospho-S287 antibody (Fig. S3d). These results verified the specificity of the antibody.

We showed that CIP2A silencing remarkably downregulated phospho-S287 (Fig. 5e), while overexpression of CIP2A elevated endogenous S287-phosphorylated PKM2 and could be reversed by coexpression of B56a (Fig. 5f). We next carried out IHC staining analysis of 15 primary human lung adenocarcinoma tissue samples using a NSCLC tissue microarray (Table. S3) to examine the clinical relevance of PKM2 S287 phosphorylation. We observed that phospho-S287 predominantly localized to the cytoplasm and was hardly detected in the nucleus (Fig. $5 \mathrm{~g}$, left panel). Notably, the expression levels of PKM2 pS287 and CIP2A were correlated with each other (Fig. $5 \mathrm{~g}$, right panel). By using the Online Survival Analysis Software ${ }^{43}$ (http://kmplot.com/analysis/index.php?p=service\&cancer=lung), we found that patients with CIP2 $A^{\text {high }} / P K M 2^{\text {high }}$ had worse prognosis than patients with CIP2 $A^{\text {high }} / P K M 2^{\text {low }}$ (Fig. 5h, left), and patients with $P K M 2^{\text {high }} / C I P 2 A^{\text {high }}$ exhibited poorer clinical outcome than patients with $P K M 2^{\text {high }} / C I P 2 A^{\text {low }}$ (Fig. 5h, right).

\section{S287 is critical to PKM2 activity}

To obtain insights into the function of S287 phosphorylation of PKM2 regulated by CIP2A, we first replaced endogenous PKM2 with either shRNA-resistant HA-PKM2, the phospho-mimetic mutant S287D HA-PKM2, or S287A HA-PKM2 in H1299 cells (Fig. 5i, j). Subcellular fractionation analysis indicated that S287D PKM2 accumulated predominantly in the cytoplasm and that resistance to CIP2A depletion-induced nuclear translocation (Fig. 5i). In contrast, compared to WT HA-PKM2-expressing cells, S287A HA-PKM2 presented lower PK activity (Fig. 5j), lower OXPHOS (Fig. 5k), and higher ECAR (Fig. 
S3e), and was incapably modulated by CIP2A ectopic expression (Fig. 5j, k, Fig. S3e). Additionally, H1299 cells expressing S287A HA-PKM2 maintained more acidic culture medium regardless of CIP2A expression (Fig. S3f). These results revealed that PKM2 phosphorylation at S287 is required for the change in oligomerization, nuclear translocation, PK activation and glucose flux regulated by CIP2A.

\section{S287 facilitates S37 in modulating PKM2 nuclear localization}

We further used the other two available phospho-specific antibodies against PKM2 to determine whether it is influenced by CIP2A. In H1299 and A549 cells, we found that CIP2A depletion had no effect on the phosphorylation level of PKM2 Y105 but significantly increased S37 phosphorylation and downregulated S287 phosphorylation (Fig. 5e). We tested whether these observations were associated with PP2A, and found that ectopically expressed CIP2A reduced phospho-S37 and increased phospho-S287 and was reversed by coexpression of B56a (Fig. 5f); conversely, depletion of the PP2A A subunit blocked CIP2A silencing-induced phospho-S37 upregulation and phospho-S287 downregulation (Fig. 6a). These results seemed inconsistent with the fact that CIP2A inhibits PP2A-mediated serine/threonine dephosphorylation and prompted us to explore whether there might be crosstalk between the phosphorylation of S37 and S287.

Previous studies indicated that the phosphorylation of PKM2 at S37 was ERK1/2 dependent and could promote PKM2 binding to importin a5, and translocated to the nucleus ${ }^{32}$. Interestingly, crystal structure analysis (PDB code 3SRD) revealed that the ERK1/2 binding sites (Ile 429/Leu 431) and the pivotal residues Arg399/400 of the nuclear localization signal (NLS) sequence, which bind to importin a5, are buried in the prominent C-C interface (Fig. 6b), whereas CIP2A-induced tetramerization may obstruct accessibility to PKM2, and the dimer stage of PKM2 is likely exposed to recruit ERK1/2 and importin a5, which promote S37 phosphorylation and nuclear PKM2 translocation. We found that ectopic CIP2A expression caused a clear reduction of the binding of HA-PKM2 to endogenous ERK1/2 and importin a5, whereas the HA-PKM2 S287A mutant, which presented in a dimer state, reinforced this interaction and was largely unaffected by transfection with Flag-CIP2A (Fig. 6C). Consistent with previous described ${ }^{32}$, S37A PKM2 failed to transport into the nucleus (Fig. 6d); surprisingly, S37A mutant also abrogated the PKM2 nuclear translocation induced by CIP2A knockdown (Fig. 6d). These results suggested that both S287 and S37 are required for nuclear translocation of PKM2 caused by CIP2A depletion.

\section{Modification of PKM2 S287 controls cell proliferation}

We then examined the role of PKM2 S287 phosphorylation on cell proliferation, and found that compared to cells expressing WT PKM2, cells expressing S287A PKM2 exhibited enhanced, while cells expressing phosphorylation mimetic S287D PKM2 showed reduced cell proliferation (Fig 7a). Consistent with these observations, we found by the three-dimensional cell culture assays seeding the same number of cells, that H1299 cells expressing S287A PKM2 presented the greatest number of tumor spheroids compared with cells expressing WT and S287D transcripts of PKM2 (Fig.7b). Notably, HA-PKM2 S287A mutant enhanced the expression of Myc and phosphorylation of STAT3 in H1299 cells (Fig. 7c). 
Given that PKM2 S287 dephosphorylation displays a significant growth advantage and may counteract the tumor suppression effect of CIP2A inhibition, PKM2 activation and Warburg effect inhibition could potentiate the tumor-suppressive effect of CIP2A depletion. We tested this possibility and found that in H1299 cells expressing WT PKM2, CIP2A silencing significantly increased sensitivity of the cells to TEPP46 and a glycolysis inhibitor 2-DG, but no such phenomena were observed in S287D cells (Fig. 7d), supporting that S287 dephosphorylation restricts the tumor suppressive effect of CIP2A deficiency.

\section{Combinatory modifications of CIP2A and PKM2 inhibits tumor growth in vivo}

As an alternative approach for depleting CIP2A, we induced CIP2A degradation using celastrol, a small natural compound previously discovered by our group that can trigger proteasomal degradation of CIP2A with the carboxyl terminus of Hsp70-interacting protein (CHIP) as the E3 ligase ${ }^{19}$. We showed that in celastrol-treated cells, CIP2A and p-S287 PKM2 were downregulated while p-S37 PKM2 was upregulated, in a dose-dependent manner, although the PKM2 protein was not changed (Fig 7e). We next tested the combined effect of celastrol and TEPP-46 or 2-DG, and found that both TEPP-46 and 2-DG enhanced the inhibitory effect of celastrol on proliferation of NSCLC cells (Fig. 7f, left panel), and a Bliss synergism analysis verified the synergistic effects of the two combination strategies (Fig. 7f, lower right panel). In addition, the crystal violet staining experiment revealed that dual treatment of A549 and H1299 cells with TEPP-46 $(30 \mu \mathrm{M}) /$ celastrol or 2-DG $(25 \mathrm{mM}) /$ celastrol $(1 \mu \mathrm{M})$ substantially suppressed the clonogenic growth and that the combinatory regimens showed much stronger effects than each single agent treatment (Fig. 7g).

We next examined the in vivo antitumor efficiency of this combination treatment in a xenograft model. We observed that monotherapy with either celastrol, TEPP-46 or 2-DG had a modest effect on tumor growth (Fig. 8a-C) and did not affect body weight of the mice (Fig. 8d). Monotherapy using these agents inhibited tumor cell proliferation, as indicated by Ki67, compared to the controls (Fig. 8e). Importantly, celastrol/TEPP-46 and celastrol/2-DG combinatory regimens exhibited substantially enhanced inhibition on tumor growth (Fig.8a-c) and suppression of cell proliferation reflected by the expression level of Ki67 (Fig. 8e), compared to each monotherapy. We found that in tumor tissues isolated from mice treated with celastrol alone or celastrol-based combination regimens, CIP2A and p-S287 PKM2 were substantially inhibited (Fig. 8e). The results indicated that PKM2 activation and glycolysis inhibition were able to synergize with CIP2A-targeted therapy in suppressing NSCLC cell proliferation in vivo.

\section{Discussion}

Although the past few decades have witnessed enormous progress in the understanding of metabolic reprogramming in cancer, the molecular mechanisms for alterations towards aerobic glycolysis in NSCLC remain unclear. Here, we demonstrated that CIP2A simultaneously impinges PKM2 in terms of both metabolic and nonmetabolic (nuclear) functions through inhibition of the PP2A regulatory B56a subunit. On the one hand, CIP2A stabilizes the oligomerization of PKM2 and promotes its PK activity; on the other hand, phosphorylation of PKM2 S287 induced by CIP2A fosters tetramer formation and hinders the 
interaction with NLS and downstream S37 phosphorylation for PKM2 nuclear translocation. Hence, CIP2A-depleted cells show a preference for glycolysis, and the combination of pharmacological degradation of CIP2A by celastrol with inhibition of glycolysis or PKM2 activation by specific agents could lead to a synergetic tumor suppression effect in NSCLC cellular mouse models.

Previous works including those from our group have shown that CIP2A is frequently elevated in human lung tumor tissues compared to corresponding normal tissue and is required for lung cancer cell growth and transformation ${ }^{33,34}$. Furthermore, CIP2A has been identified as a major determinant of EGFR tyrosine kinase inhibitor erlotinib-induced apoptosis in NSCLC cells without EGFR mutation ${ }^{44}$. Additionally, CIP2A/PP2A/Akt signaling was constitutively activated in erlotinib-resistant cells and could mediate drug resistance to erlotinib in EGFR-mutated NSCLC cells ${ }^{45}$. This evidence suggests that modulation of CIP2A could represent a promising approach for NSCLC treatment. However, CIP2A exerts its tumorigenic function mainly by inhibiting the phosphatase activity of PP2A, which would largely depend on the substrate of PP2A. In this study, CIP2A depletion prompted NSCLC cells to undergo aggressive glycolysis by dephosphorylating PKM2 at S287, indicating that the efficiency of CIP2Atargeted therapy could be counteracted by activating pro-oncogenic signaling. Indeed, the application of some small molecular inhibitors of PP2A to complement and sensitize chemotherapy or radiotherapy have been reported $46,47,48$.

Several reports have demonstrated that CIP2A is associated with metabolic reprogramming. Recent studies have revealed that in human retinal pigment epithelium RPE1 cells, CIP2A depletion shifts metabolism towards the glycolytic pathway probed by ECAR, and the expression of glycolysis-related genes was also elevated, whereas tricarboxylic acid (TCA) cycle-related genes were decreased ${ }^{49}$. CIP2A promotes primary cilia disassembly and CIP2A depletion induces metabolic reprogramming independent of primary cilia ${ }^{49}$. We showed that in NSCLC cells CIP2A knockdown significantly increased the glycolysis rate and lactate production while reducing OXPHOS. Another study revealed that CIP2A augments mitochondrial respiration without compromising glycolytic processes in the chronic myeloid leukemia cell line $\mathrm{K}_{562}{ }^{50}$. These observations indicated that the regulatory mechanism of CIP2A in metabolic reprogramming is complicated, and considerable work still remains to be done to understand the biological and pathological functions of CIP2A.

The activity of the metabolic enzyme PKM2 is associated with its multimerization status and cellular sublocation, which could be regulated by posttranslational modifications, including phosphorylation. Tyr105 and Ser37 are two well-explored modification sites that are stimulated by the tyrosine kinase receptor fibroblast growth factor (FGFR) and EGFR, respectively 25,32 . It has been reported that the dualspecificity phosphatase Cdc25A can readily dephosphorylate PKM2 pS37 only in the nucleus ${ }^{51}$. In this study, we first demonstrated that the PP2A regulatory subunit B56a dephosphorylates PKM2 at S287 in the cytoplasm, thus increasing the number of cytoplasmic phosphatases that regulate PKM2 activity. Because of the technical difficulty of determining the ratio of the PKM2 tetramer/dimer, the expression of CIP2A and S287 phosphorylation, which is measured by the specific antibodies, may thus serve as a 
specific sensor. Although B56 family members harbor a highly conserved substrate interaction surface-

exposed pocket ${ }^{41}, 42$, our Co-IP data showed that only B56a could bind to PKM2, while B56y and B56ع, which are also associated with CIP2A, could not. In addition, PKM2 was not found in the B56a binding protein identified by $M S^{41,42}$, suggesting that PKM2 is a new substrate of PP2A (B56a).

Glycolysis is a dominant and aggressive metabolic phenotype that can provide efficient energy supplementation to meet the requirement of rapid tumor cell anabolic growth. However, only ${ }^{18} \mathrm{~F}-\mathrm{FDG}$ $\mathrm{PET} / \mathrm{CT}$ has been used to diagnose malignant tumors, especially metastatic lesions, by evaluating glucose uptake. Currently, there are no drugs targeting aberrant glycolic pathways that have been successfully used in the clinic for the treatment of cancer. Furthermore, previous studies have reported that PKM2-induced excessive glycolysis is positively correlated with drug resistance induced by various convenient chemotherapies or targeted therapies ${ }^{52,53}$. In this study, we revealed that the glycolysis inhibitor 2-DG and PKM2 activator delayed NSCLC cell growth in vitro and in vivo, and these two agents could synergistically inhibit NSCLC cell proliferation in vitro and in vivo in combination with CIP2A shRNA for CIP2A depletion or celastrol for CIP2A degradation.

In summary, we identified CIP2A as a novel binding partner and modulator of PKM2. Mechanistically, the phosphorylation of PKM2 at the S287 site is critical for CIP2A to regulate PKM2 functions and to suppress the Warburg effect in NSCLC cells. The combination of a glycolysis inhibitor or PKM2 activator with the CIP2A-targeting compound celastrol leads to a prominent synergistic antitumor effect and could serve as a novel and promising strategy for treating patients with NSCLC (Fig. 8f).

\section{Methods}

\section{Cell culture and transfection}

Embryonic kidney HEK293T and NSCLC cell lines A549, H1299, and H1975 (the American Type Culture Collection, Manassas, VA, USA) were cultured in DMEM or RPMI 1640 medium supplemented with 10\% fetal bovine serum (HyClone, Logan, UT, USA) at $37^{\circ} \mathrm{C}$ in a $5 \% \mathrm{CO}_{2} / 95 \%$ air incubator. Transient transfection of plasmids or siRNAs was conducted using the Lipofectamine 3000 kit (Invitrogen) according to the vendor's instructions. All siRNAs were synthesized by GenePharma (Shanghai, China) and the sequences is shown in Table $\mathrm{S} 1$.

\section{Plasmids and mutagenesis}

Polymerase chain reaction (PCR)-amplified human CIP2A, B56a, B56y 1 and $B 56 \varepsilon$ were cloned into the pcDNA3.1-Flag vector. PCR-amplified human $P K M 2$ cDNA and $P K M 2$ truncated mutants were cloned into the pcs2-HA vector. GST-CIP2A was cloned into the pGEX-4T-1 vector. PKM2 mutants, including L218A/V221A, F280A/I283A, S222A, S243A, S249A, S287A, S287D and S37A, were generated using a QuikChange site-directed mutagenesis kit (Stratagene, La Jolla, CA, USA). Short hairpin RNA (shRNA)-resistant PKM2 was generated by introducing nonsense mutations in shRNA-targeting 
sites containing C1170T, C1173T, T1174C and G1176T. Core plasmids used to generate CIP2A and shRNA-resistant HA-PKM2 [wild type (WT), S287A and S287D] stable expression cell lines were constructed in pCDH-CMV-GFP and pLVX-IRES-Neo vectors, respectively.

\section{Virus packaging and stable cell line generation}

The shRNAs were cloned into the pLKO.1 vector. The target sequences were listed in Supplementary Table. S1. The shRNA gene knockdown or open reading frame gene expression lentiviral vectors were transfected into 293T cells together with packaging plasmid (psPAX2) and envelope plasmid (pMD2G) using the Lipo3000 kit (Invitrogen) according to the manufacturer's instructions. After 36 hours and 60 hours, the viruses were collected, filtered, and used to infect target cells in the presence of 8 $\mu \mathrm{g} / \mathrm{mL}$ polybrene for 12 hours. The infected cells were selected by puromycin or $\mathrm{G} 418$ and evaluated by western blotting.

\section{Seahorse metabolic assays}

A549 or H1299 cells were seeded into an XF96 cell culture plate (Seahorse Bioscience) at a density of $1 \times 10^{4}$ cells per well and incubated with complete medium overnight. Extracellular acidification rate (ECAR) and oxygen consumption rate (OCR) were detected by a Seahorse XFe-96 Analyser (Agilent, Cheadle, Greater Manchester, UK). To monitor changes in ECAR, 10 mM glucose, $1.0 \mu \mathrm{M}$ oligomycin and 50 mM 2-deoxyglucose (2-DG) were used. Changes in OCR were assessed by sequential injection of final concentrations of $1.0 \mu \mathrm{M}$ oligomycin, $0.5 \mu \mathrm{M}$ FCCP and $0.5 \mu \mathrm{M}$ rotenone and antimycin A. Corresponding metabolic parameters were further calculated by WAVE Software (Seahorse Bioscience, Agilent) and normalized based on the cell number of each well.

\section{Mass spectrometry (MS)}

The CIP2A complex was obtained by immunoprecipitation (IP) with anti-CIP2A from a 15-cm culture dish of A549 and H1299 cells. Cells were lysed in IP buffer [40 mM Tris· Cl (pH 7.4), $150 \mathrm{mM} \mathrm{NaCl,} 1.5 \mathrm{mM}$ MgCl2, 0.5\% nonidet P-40, 2 mM EDTA, and 1 mM PMSF, 1 mM proteinase inhibitor cocktail] and immunoprecipitated with Protein A/G PLUS-Agarose overnight at $4{ }^{\circ} \mathrm{C}$. The beads were washed five times with IP buffer. The eluted proteins were resolved by SDS-PAGE, silver stained, and the gel bands of proteins were excised for in-gel digestion, and the proteins were identified using MS. Briefly, proteins were treated with $25 \mathrm{mM}$ dithiothreitol (DTT) and alkylated with $55 \mathrm{mM}$ iodoacetamide to reduce disulfide. Sequencing grade-modified trypsin in 50 mM ammonium bicarbonate was used for in-gel digestion at 37 ${ }^{\circ} \mathrm{C}$ overnight. The peptides were extracted twice with $1 \%$ trifluoroacetic acid in $50 \%$ acetonitrile aqueous solution for $30 \mathrm{~min}$. To reduce the volume, the peptide extracts were centrifuged in a SpeedVac. For LCMS/MS analysis, peptides were separated by 60 min gradient elution at a flow rate of $0.3 \mu \mathrm{L} / \mathrm{min}$ with a Thermo-Dionex Ultimate 3000 HPLC system, which was directly interfaced with a Thermo LTQ-Orbitrap Velos pro mass spectrometer. 
PKM2 posttranscriptional modification sites were analyzed by LC-MS/MS. Briefly, H1299 cells were cotransfected with Flag-CIP2A and HA-PKM2 for $48 \mathrm{~h}$, and cell lysates immunoprecipitated by HA magnetic beads (CST) were subjected to $10 \%$ SDS-PAGE and visualized by Coomassie blue staining. The stained bands at a molecular weight of $58 \mathrm{kDa}$ were excised and revealed by LC-MS/MS at Tsinghua University.

\section{Immunoprecipitation, GST pull-down, and western blot assays}

Cell lysates used for the immunoprecipitation (IP) assay were extracted in IP lysis buffer at $4{ }^{\circ} \mathrm{C}$ for 30 minutes. Supernatants were clarified by centrifugation at top speed $(16,000 \times \mathrm{g})$ for $10 \mathrm{~min}$ at $4^{\circ} \mathrm{C}$. The supernatants were incubated with the corresponding antibodies ( $2 \mu \mathrm{g}$; Table S2) as indicated and $30 \mu \mathrm{L}$ of Protein A/G PLUS-Agarose (Santa Cruz Biotechnology) at $4{ }^{\circ} \mathrm{C}$ overnight. The beads were washed five times with IP lysis buffer. The beads were boiled with $2 \times$ SDS loading buffer for 6 min. The input lysates and immunoprecipitate were separated by $5 \sim 15 \%$ SDS/PAGE and western blotting assays. Recombinant GST-CIP2A proteins were produced in Escherichia coli BL21 (DE3) cells and purified with Glutathione Sepharose 4B (GE healthcare). GST (10 $\mu \mathrm{g})$ or GST fusion proteins were incubated with Glutathione Sepharose $4 \mathrm{~B}$ for $2 \mathrm{~h}$ at $4{ }^{\circ} \mathrm{C}$, followed by incubation with cell extracts at $4{ }^{\circ} \mathrm{C}$ overnight.

After centrifugation at $16,000 \mathrm{~g}$, the supernatants were collected as input, and the agarose beads were extensively washed five times each with $1 \mathrm{ml}$ lysis buffer, boiled with SDS loading buffer for $5 \mathrm{~min}$ and analyzed by western blotting. Nuclear and cytosolic fractions were separated using a Minute ${ }^{\mathrm{TM}}$ Cytoplasmic and Nuclear Extraction Kit for Cells (SC-003, Invent Biotechnologies) according to the manufacturers' protocol. Detailed information of the antibodies used in this study are exhibited in Table S2, and a PKM2 phosphorylation (S287)-specific antibody was generated by immunizing rabbits with S287-phosphopeptide (ILEAS(p)DGI) at ABclonal, Inc.

\section{Immunofluorescence (IF) staining and confocal microscopy}

Cells were seeded on cover slides, fixed with $4 \%$ formaldehyde, and then permeabilized with $0.3 \%$ Triton X-100/PBS at room temperature. Then, the cells were blocked in $5 \%$ bovine serum albumin/PBS for 30 min at room temperature and then incubated with primary antibody overnight at $4{ }^{\circ} \mathrm{C}$, followed by incubation with FITC/PE-labelled secondary antibodies for $1.5 \mathrm{~h}$ at room temperature. After incubation with 4',6-diamidino-2-phenylindole (DAPI) to stain the nuclei, the cells were visualized with a confocal laser scanning microscope (Nikon, N-STORM Super-Resolution). Subcellular fractionation was analyzed with the line profile tool in the LSM 780 META ZEN 2011 software package (Carl Zeiss).

\section{Sizeexclusion chromatography}

The size-exclusion chromatography (gel filtration) column (Superdex 200 Increase 10/300 GL, GE healthcare) was washed with distilled water and equilibrated with PBS. Cells were lysed in lysis buffer [50 $\mathrm{mM}$ Tris ( $\mathrm{pH}$ 7.5), $150 \mathrm{mM} \mathrm{NaCl}, 0.3 \%$ nonidet P-40, 1 mM PMSF, $1 \mathrm{mM}$ proteinase inhibitor cocktail (Roche)], and $5-7 \mathrm{mg} / \mathrm{mL}$ total protein $(500 \mu \mathrm{l})$ was loaded into the column and eluted with elution buffer phosphate buffered saline [50 mM sodium phosphate, $0.15 \mathrm{M} \mathrm{NaCl}(\mathrm{pH}$ 7.2)]. The speed rate of 
flow was $0.5 \mathrm{ml} / \mathrm{min}$. Every $300 \mu \mathrm{L}$ fraction was collected, and $20 \mu \mathrm{L}$ of each fraction was analyzed by western blot analysis.

\section{Protein crosslinking assay}

A total of $1 \times 10^{6}$ cells were lysed with sodium phosphate buffer $(\mathrm{pH} 7.3)$ containing $0.5 \%$ Triton $\mathrm{X}-100,1$ $\mathrm{mM}$ PMSF and $1 \mathrm{mM}$ proteinase inhibitor cocktail for $30 \mathrm{~min}$ at $4{ }^{\circ} \mathrm{C}$. Crude cell lysates were clarified by centrifugation at top speed $(16,000 \times \mathrm{g})$ for $15 \mathrm{~min}$ at $4{ }^{\circ} \mathrm{C}$. To carry out crosslinking reactions, the supernatants $\left(>5 \mathrm{mg} / \mathrm{mL}\right.$ ) were treated with $0.01 \%$ glutaraldehyde for 9 min at $37^{\circ} \mathrm{C}$. The reactions were terminated by adding $1 \mathrm{M}$ Tris buffer to a final concentration of $50 \mathrm{mM}$ Tris $\mathrm{Cl}(\mathrm{pH} 8.0)$. Samples were then separated by $5 \sim 15 \%$ SDS/PAGE and analyzed by western blotting.

\section{Measurement of pyruvate kinase (PK) activity}

Whole-cell PK activity was measured with a colorimetric-based PK activity assay kit (Biovision) according to the manufacturer's protocol. For the in vitro PK activity assay, PKM2 $(5 \mathrm{nM})$ was coincubated with various concentrations of GST-CIP2A $(0-600 \mathrm{nM})$. The kinase activity was measured with a colorimetricbased PK activity assay kit (Biovision) according to the manufacturer's protocol.

\section{Cell proliferation and viability}

Cells expressing different HA-PKM2 mutants were seeded into 12-well plates, and the cell confluence area was monitored every 4 hours over a 3-day period using an IncuCyte Live-Cell Analysis System (Essen Bioscience). To determine cell viability, cells infected with or without CIP2A siRNA were plated into 96well plates, and cytotoxic activity was assessed after the indicated drug treatment for $48 \mathrm{~h}$ using a Cell Counting Kit-8 (CCK-8; Vazyme) assay. The effects of indicated compounds (Table S2) on the cells were detected by CCK-8 assay, and the potential synergistic interaction of the two compounds was calculated by the Bliss model using Combenefit software ${ }^{54}$. For colony forming activity, the cells were plated into 12-well plates and treated with TEPP-46 and/or 2-DG for $48 \mathrm{~h}$, and fixed with $4 \%$ paraformaldehyde and stained with $0.1 \%$ crystal violet.

\section{Xenograft studies}

The animal studies were approved by and conducted according to the Ethics Committee of Cancer Hospital, Chinese Academy of Medical Sciences. Five-week-old female nude BALB/c mice were purchased from Vital River Laboratory Animal Technology Co., Ltd. (Beijing, China), kept in a specific pathogen-free (SPF) environment and used for the animal assays. After 7 days of acclimatization, $5 \times$ $10^{6} \mathrm{~A} 549$ cells were subcutaneously inoculated into the dorsal flank. When reaching approximately 100 $\mathrm{mm}^{3}$, the tumour-bearing mice were randomly divided into six groups (six mice per group) for treatment with the control, celastrol (2 mg/kg orally daily), TEPP-46 (100 mg/kg orally daily), 2-DG $(500 \mathrm{mg} / \mathrm{kg} / \mathrm{day}$, intraperitoneal injection), and combination treatment of celastrol with TEPP46 or 2-DG. Celastrol and TEPP-46 were dissolved in 10\% DMSO and 90\% CMC-Na (Sigma), and 2-DG was dissolved in normal 
saline.tumorvolumes were measured every 3 days by electronic calliper and calculated following the formula: volume $=1 / 2$ length $\times$ width $^{2}$. Mouse body weights were monitored regularly throughout the course of the animal experiments. Thirty-five days later, the mice were sacrificed, and thetumortissues were dissected, photographed, weighed and fixed in $4 \%$ formaldehyde for further immunohistochemistry (IHC) studies.

\section{IHC and human lung cancer tissue array}

Human lung cancer tissue arrays were purchased from Shanghai Outdo Biotech (catalogue no. HLugAde030PG-01), and the samples were obtained from patients (Table S3) with written informed consent. IHC staining of resected mouse tumor tissues and human lung cancer tissue arrays was carried out according to the manufacturer's protocol (Zhongshan Golden Bridge, Beijing, China). The quantification of IHC was calculated as IRS (0 12) = SP (0 1: $0 \%$ 25\%; $2: 26 \% \sim 50 \% ; 3: 51 \% \sim 75 \%$ and $4:>75 \%) \times \mathrm{SI}$ (0: no signal; 1 : weak; 2 : moderate and 3: strong), where IRS is the immunoreactivity score, SP is the proportion of stained positive cells and SI is the staining intensity. Detailed information about the antibodies used in IHC are listed in Table. S2.

\section{Statistical analysis}

All experiments were performed at least three times, and the results are presented as the mean \pm SD. Differences between data groups were evaluated for significance using Student's $t$ test of unpaired data or one-way analysis of variance. $P<0.05$ (two-sided) was considered statistically significant (* indicates $P<0.05$, ** indicates $P<0.01$ and $* * *$ indicates $P<0.001 ;$ n.s. indicates not statistically significant).

\section{Declarations}

\section{Authors' contributions}

GBZ conceived the study and designed the experiments. LJL, DW, YFZ, FYY, DWX, HY, ZW, BBS, SHG, HG, $X C Z$, and GZW performed the experiments. LJL, YFZ and GBZ analyzed the results. LJL and GBZ wrote the manuscript. GBZ supervised this study.

\section{Acknowledgements}

This work was supported by the National Key Research and Development Program of China (No. 2020YFA0803300), the Key Project of the National Natural Science Foundation of China (81830093), the CAMS Innovation Fund for Medical Sciences (CIFMS; Nos. 2021-RC310-003, 2020-RC310-002), CAMS Initiative for Innovative Medicine (2021-1-I2M-012), and the National Natural Science Foundation of China (82073092 and 81802796).

\section{Data availability}


All the data supporting the findings of this study are available within this paper and its supplementary information.

\section{Additional information}

Supplementary information includes two tables and eight figures.

\section{Competing interests}

The authors declare that they have no competing interests.

\section{References}

1. Sung $\mathrm{H}$, et al. Global Cancer Statistics 2020: GLOBOCAN Estimates of Incidence and Mortality Worldwide for 36 Cancers in 185 Countries. CA Cancer J Clin 71, 209-249 (2021).

2. Tian X, Gu T, Lee MH, Dong Z. Challenge and countermeasures for EGFR targeted therapy in nonsmall cell lung cancer. Biochimica et biophysica acta Reviews on cancer 1877, 188645 (2021).

3. Horn L, et al. First-Line Atezolizumab plus Chemotherapy in Extensive-Stage Small-Cell Lung Cancer. N Engl J Med 379, 2220-2229 (2018).

4. Garon EB, et al. Pembrolizumab for the treatment of non-small-cell lung cancer. N Engl J Med 372, 2018-2028 (2015).

5. Horn L, et al. Nivolumab Versus Docetaxel in Previously Treated Patients With Advanced Non-SmallCell Lung Cancer: Two-Year Outcomes From Two Randomized, Open-Label, Phase III Trials (CheckMate 017 and CheckMate 057). J Clin Oncol 35, 3924-3933 (2017).

6. Duma N, Santana-Davila R, Molina JR. Non-Small Cell Lung Cancer: Epidemiology, Screening, Diagnosis, and Treatment. Mayo Clinic proceedings 94, 1623-1640 (2019).

7. Soo Hoo L, Zhang JY, Chan EK. Cloning and characterization of a novel 90 kDa 'companion' autoantigen of p62 overexpressed in cancer. Oncogene 21, 5006-5015 (2002).

8. Junttila MR, et al. CIP2A inhibits PP2A in human malignancies. Cell 130, 51-62 (2007).

9. Khanna A, Pimanda JE. Clinical significance of cancerous inhibitor of protein phosphatase $2 \mathrm{~A}$ in human cancers. International journal of cancer 138, 525-532 (2016).

10. Chen KF, et al. CIP2A mediates effects of bortezomib on phospho-Akt and apoptosis in hepatocellular carcinoma cells. Oncogene 29, 6257-6266 (2010).

11. Lucas CM, et al. High CIP2A levels correlate with an antiapoptotic phenotype that can be overcome by targeting BCL-XL in chronic myeloid leukemia. Leukemia 30, 1273-1281 (2016).

12. Nagelli S, Laine A, Suomi T, Elo L, Westermarck J. 43P - CIP2A as a novel target to combat basal like breast cancer. Annals of Oncology 30, iii14-iii15 (2019).

13. Laine $A$, et al. Senescence sensitivity of breast cancer cells is defined by positive feedback loop between CIP2A and E2F1. Cancer Discovery 3, 182-197 (2013). 
14. Liu C-Y, et al. Targeting SET to restore PP2A activity disrupts an oncogenic CIP2A-feedforward loop and impairs triple negative breast cancer progression. EBioMedicine 40, 263-275 (2019).

15. Kerosuo L, et al. Enhanced expression of MycN/CIP2A drives neural crest toward a neural stem celllike fate: Implications for priming of neuroblastoma. Proc Natl Acad Sci U S A 115, E7351-e7360 (2018).

16. Laine A, et al. CIP2A Interacts with TopBP1 and Drives Basal-Like Breast Cancer Tumorigenesis. Cancer research 81, 4319-4331 (2021).

17. Kim JS, Kim EJ, Oh JS, Park IC, Hwang SG. CIP2A Modulates Cell-Cycle Progression in Human Cancer Cells by Regulating the Stability and Activity of Plk1. Cancer Research 73, 6667-6678 (2013).

18. Yang $X$, et al. Inhibition of CIP2A attenuates tumor progression by inducing cell cycle arrest and promoting cellular senescence in hepatocellular carcinoma. Biochem Biophys Res Commun 495, 1807-1814 (2018).

19. Liu Z, et al. Cancerous inhibitor of PP2A (CIP2A) is targeted by natural compound celastrol for degradation in non-small-cell lung cancer. Carcinogenesis 35, 905-914 (2014).

20. Martínez-Reyes I, Chandel NS. Cancer metabolism: looking forward. Nat Rev Cancer 21, 669-680 (2021).

21. Noguchi T, Inoue H, Tanaka T. The M1- and M2-type isozymes of rat pyruvate kinase are produced from the same gene by alternative RNA splicing. J Biol Chem 261, 13807-13812 (1986).

22. Gao X, Wang H, Yang JJ, Liu X, Liu ZR. Pyruvate kinase $M 2$ regulates gene transcription by acting as a protein kinase. Molecular cell 45, 598-609 (2012).

23. Yang W, et al. Nuclear PKM2 regulates $\beta$-catenin transactivation upon EGFR activation. Nature 480, 118-122 (2011).

24. Luo W, et al. Pyruvate kinase M2 is a PHD3-stimulated coactivator for hypoxia-inducible factor 1 . Cell 145, 732-744 (2011).

25. Hitosugi T, et al. Tyrosine phosphorylation inhibits PKM2 to promote the Warburg effect and tumor growth. Sci Signal 2, ra73 (2009).

26. Lv L, et al. Acetylation targets the $\mathrm{M} 2$ isoform of pyruvate kinase for degradation through chaperonemediated autophagy and promotes tumor growth. Mol Cell 42, 719-730 (2011).

27. Anastasiou D, et al. Inhibition of pyruvate kinase $M 2$ by reactive oxygen species contributes to cellular antioxidant responses. Science 334, 1278-1283 (2011).

28. Liu F, et al. PKM2 methylation by CARM1 activates aerobic glycolysis to promote tumorigenesis. Nature cell biology 19, 1358-1370 (2017).

29. Chaneton $B$, et al. Serine is a natural ligand and allosteric activator of pyruvate kinase M2. Nature 491, 458-462 (2012).

30. Keller KE, Tan IS, Lee YS. SAICAR stimulates pyruvate kinase isoform M2 and promotes cancer cell survival in glucose-limited conditions. Science 338, 1069-1072 (2012). 
31. Lv L, et al. Mitogenic and oncogenic stimulation of K433 acetylation promotes PKM2 protein kinase activity and nuclear localization. Molecular cell 52, 340-352 (2013).

32. Yang W, et al. ERK1/2-dependent phosphorylation and nuclear translocation of PKM2 promotes the Warburg effect. Nature cell biology 14, 1295-1304 (2012).

33. Liu Z, Ma L, Wen ZS, Cheng YX, Zhou GB. Ethoxysanguinarine Induces Inhibitory Effects and Downregulates CIP2A in Lung Cancer Cells. ACS Med Chem Lett 5, 113-118 (2014).

34. Ma L, et al. Overexpression and small molecule-triggered downregulation of CIP2A in lung cancer. PLoS One 6, e20159 (2011).

35. Morita M, et al. PKM1 Confers Metabolic Advantages and Promotes Cell-Autonomous Tumor Cell Growth. Cancer cell 33, 355-367 e357 (2018).

36. Ning $X$, et al. Discovery of novel naphthoquinone derivatives as inhibitors of the tumor cell specific M2 isoform of pyruvate kinase. Eur J Med Chem 138, 343-352 (2017).

37. Wang HJ, et al. JMJD5 regulates PKM2 nuclear translocation and reprograms HIF-1 a-mediated glucose metabolism. Proceedings of the National Academy of Sciences of the United States of America 111, 279-284 (2014).

38. Anastasiou D, et al. Pyruvate kinase M2 activators promote tetramer formation and suppress tumorigenesis. Nature chemical biology 8, 839-847 (2012).

39. Shi Y. Serine/threonine phosphatases: mechanism through structure. Cell 139, 468-484 (2009).

40. Wang $\mathrm{J}$, et al. Oncoprotein CIP2A is stabilized via interaction with tumor suppressor PP2A/B56. EMBO Rep 18, 437-450 (2017).

41. Hertz EPT, et al. A Conserved Motif Provides Binding Specificity to the PP2A-B56 Phosphatase. Molecular cell 63, 686-695 (2016).

42. Wu CG, et al. PP2A-B' holoenzyme substrate recognition, regulation and role in cytokinesis. Cell discovery 3, 17027 (2017).

43. Gyorffy B, Surowiak P, Budczies J, Lanczky A. Online Survival Analysis Software to Assess the Prognostic Value of Biomarkers Using Transcriptomic Data in Non-Small-Cell Lung Cancer. PLoS ONE 8, e82241 (2013).

44. Wang CY, et al. CIP2A mediates erlotinib-induced apoptosis in non-small cell lung cancer cells without EGFR mutation. Lung Cancer 85, 152-160 (2014).

45. Saafan $\mathrm{H}$, et al. Constitutive Cell Proliferation Regulating Inhibitor of Protein Phosphatase 2A (CIP2A) Mediates Drug Resistance to Erlotinib in an EGFR Activating Mutated NSCLC Cell Line. Cells 10, (2021).

46. Mirzapoiazova T, et al. Protein Phosphatase 2A as a Therapeutic Target in Small Cell Lung Cancer. Mol Cancer Ther, (2021).

47. O'Connor CM, Perl A, Leonard D, Sangodkar J, Narla G. Therapeutic targeting of PP2A. The international journal of biochemistry \& cell biology 96, 182-193 (2018). 
48. Ho WS, et al. LB-100, a novel Protein Phosphatase 2A (PP2A) inhibitor, sensitizes malignant meningioma cells to the therapeutic effects of radiation. Cancer letters 415, 217-226 (2018).

49. Jeong AL, et al. Oncoprotein CIP2A promotes the disassembly of primary cilia and inhibits glycolytic metabolism. EMBO Rep 19, (2018).

50. Austin JA, et al. Cancerous inhibitor of protein phosphatase 2A (CIP2A) modifies energy metabolism via 5 ' AMP-activated protein kinase signalling in malignant cells. The Biochemical journa/ 476, 2255-2269 (2019).

51. Liang J, et al. PKM2 dephosphorylation by Cdc25A promotes the Warburg effect and tumorigenesis. Nat Commun 7, 12431 (2016).

52. Wong TL, et al. CRAF Methylation by PRMT6 Regulates Aerobic Glycolysis-Driven Hepatocarcinogenesis via ERK-Dependent PKM2 Nuclear Relocalization and Activation. Hepatology (Baltimore, Md) 71, 1279-1296 (2020).

53. Li Q, et al. Nuclear PKM2 contributes to gefitinib resistance via upregulation of STAT3 activation in colorectal cancer. Scientific reports 5, 16082 (2015).

54. Di Veroli GY, et al. Combenefit: an interactive platform for the analysis and visualization of drug combinations. Bioinformatics 32, 2866-2868 (2016).

\section{Figures}


a

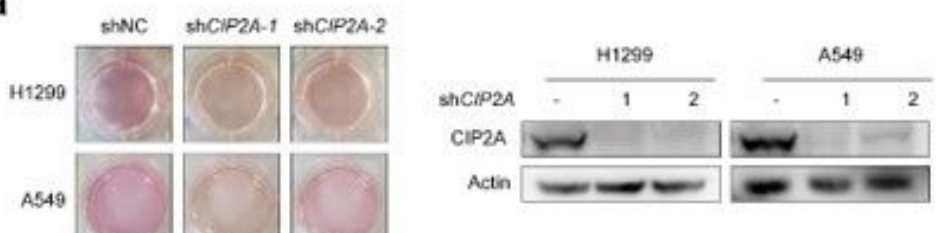

b

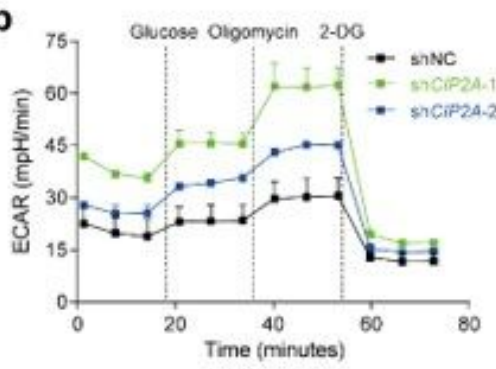

C

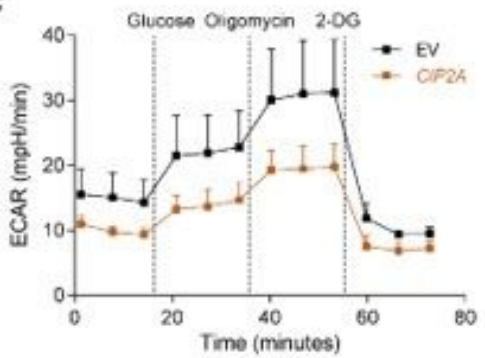

d

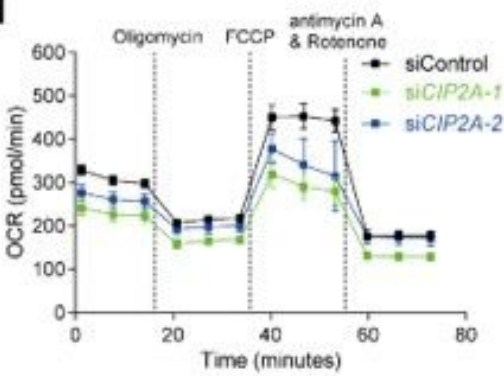

e

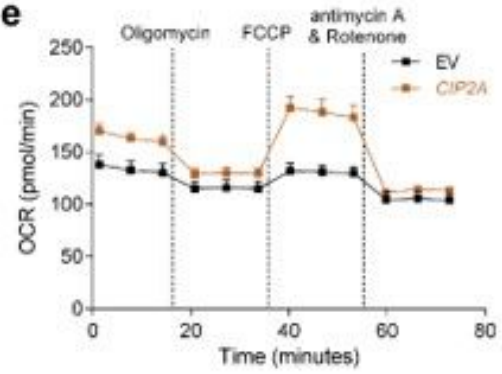

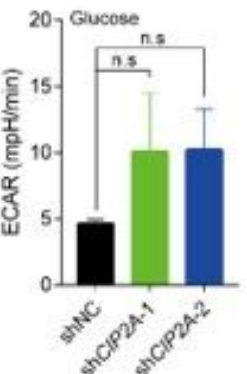
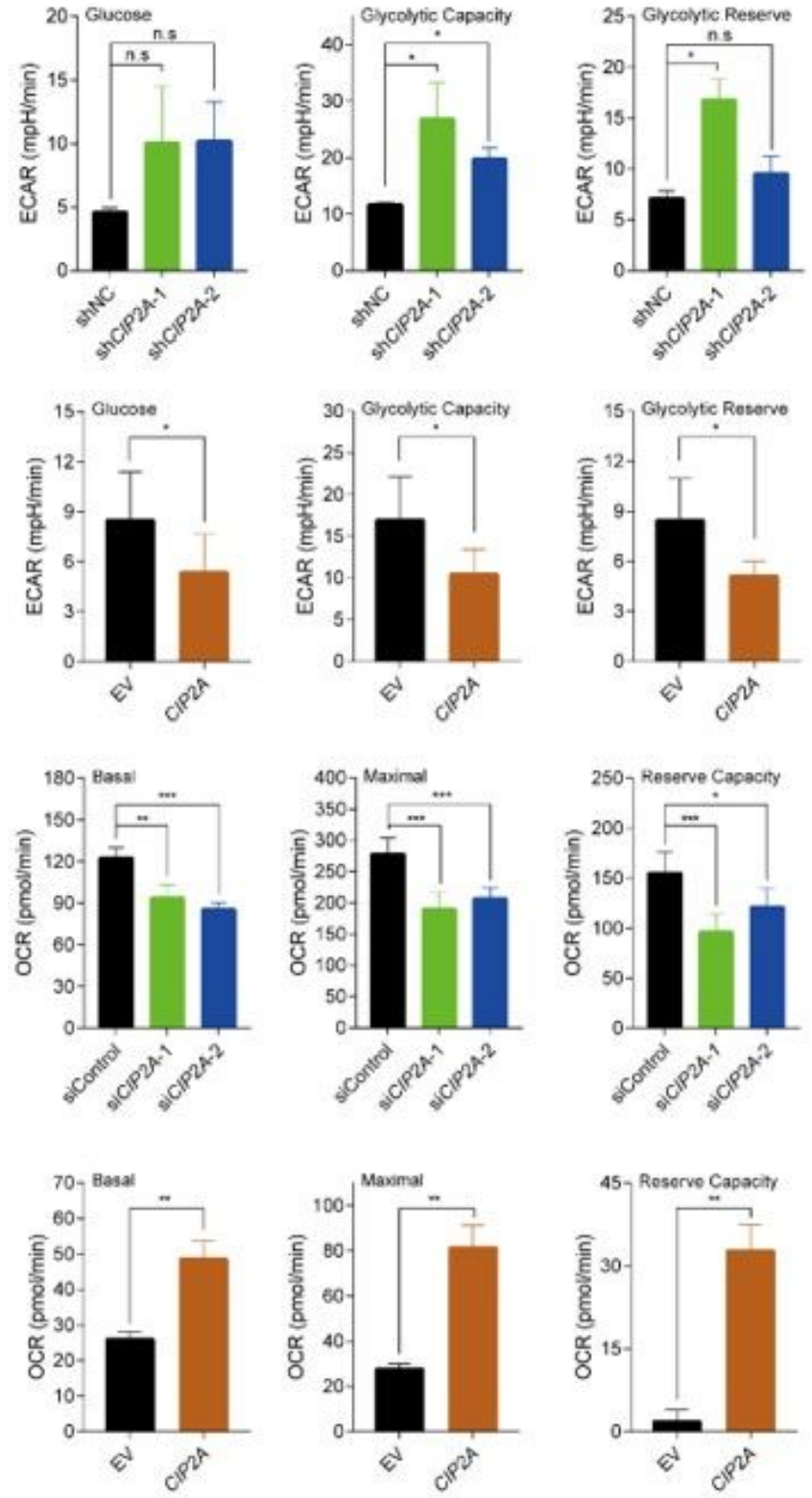

Fig. 1

Figure 1

CIP2A modulates the extracellular acidification rate (ECAR) and the oxygen consumption rate (OCR) in NSCLC cells. (a) Representative bright-field images of cell culture media of $\mathrm{H} 1299$ and A549 cells that were transfected with siCIP2A. (b, c) ECAR of $\mathrm{H} 1299$ cells that were transfected with siCIP2A (b) or pCDHCMV-CIP2A (c), analyzed with a Seahorse XFe96 analyzer. n.s., not significant for the indicated comparison, ${ }^{*} P<0.05,{ }^{*} P<0.01$, compared with shNC cells (unpaired Student's $t$ test). (d, e) OCR of 
$\mathrm{H} 1299$ cells that were transfected with siCIP2A (d) or pCDH-CMV-CIP2A (e), analyzed with a Seahorse XFe96 analyzer. Student's $t$ test, $* P<0.05, * \star P<0.01, * \star \star P<0.001$.

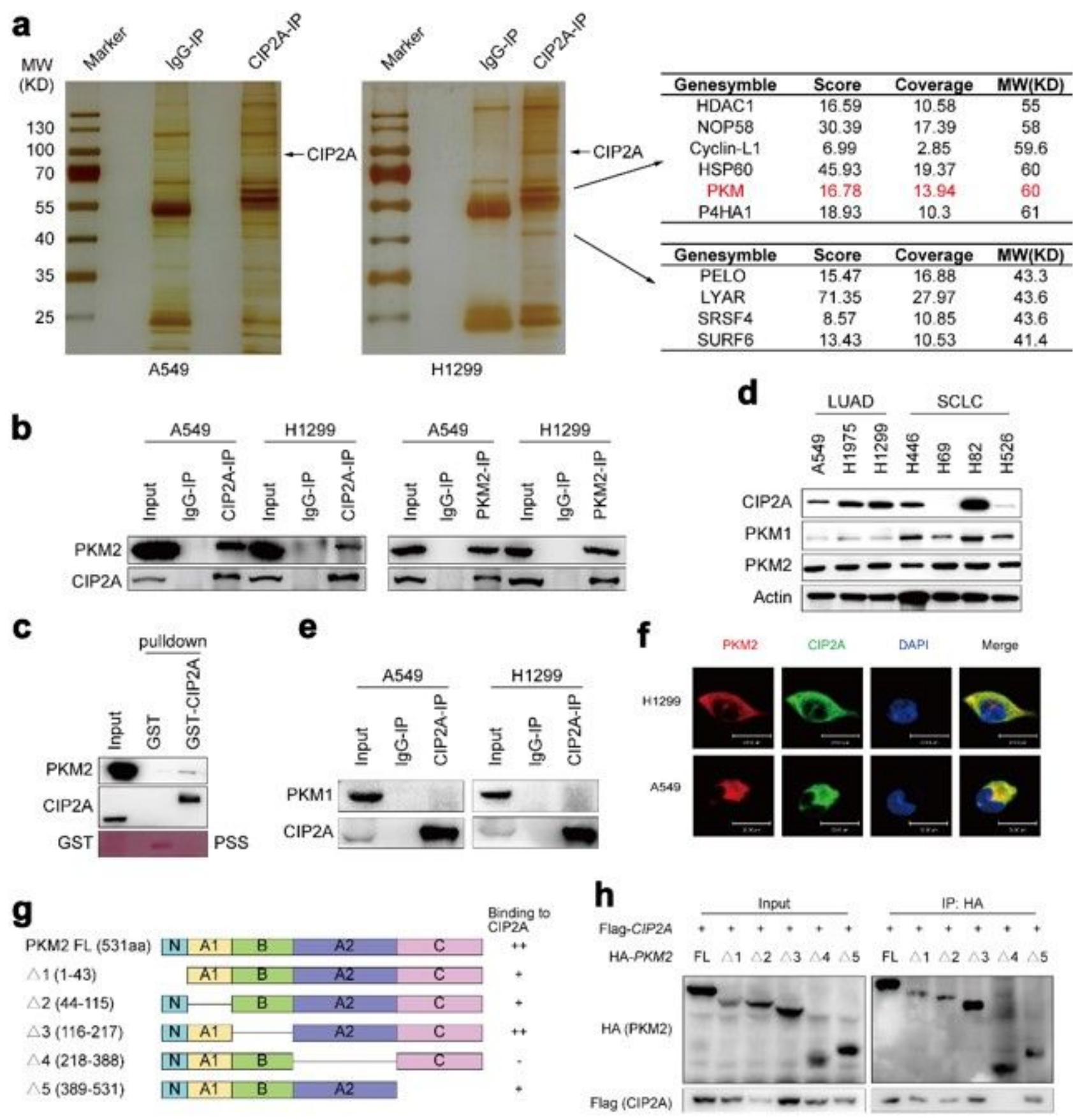

Fig. 2

Figure 2

CIP2A physically interacts with PKM2. (a) Mass spectrum analysis of CIP2A-interacting proteins. Cellular extracts were prepared from A549 and H1299 cells, followed by immunoprecipitation with an anti-CIP2A antibody. The distinct protein bands on silver-stained SDS/PAGE gels were retrieved and analyzed by mass spectrometry. (b) IP and immunoblotting assays using the indicated antibodies and cell lysates. (c) 
GST or GST-CIP2A was incubated with $\mathrm{H} 1299$ cell lysates in vitro to detect endogenous PKM2 using the indicated antibodies. PSS, Ponceau-S stain. (d) The expression of CIP2A, PKM1 and PKM2 was detected by western blot in the indicated cells. (e) Interaction between endogenous PKM1 and CIP2A proteins was detected by immunoprecipitation assay followed by western blot using the anti-CIP2A antibody in A549 and H1299 cells. (f) Immunofluorescence assays of H1299 and A549 cells using antibodies against PKM2 (red) and CIP2A (green), and 4',6-Diamidino-2-phenylindole (DAPI) to stain nuclei (blue). Scale bars, $30 \mu \mathrm{m}$. (g) Schematic representing PKM2 protein fragments used to identify the CIP2A binding region in PKM2. (h) HA-tagged PKM2 mutants and Flag-tagged CIP2A were co-transfected into 293T cells, which were analyzed for Co-IP and subsequent western blotting. IP, immunoprecipitation. 


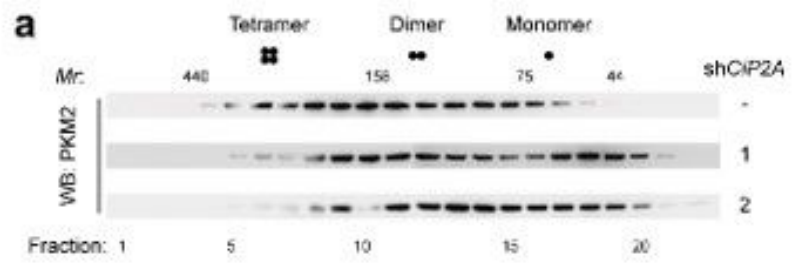

C

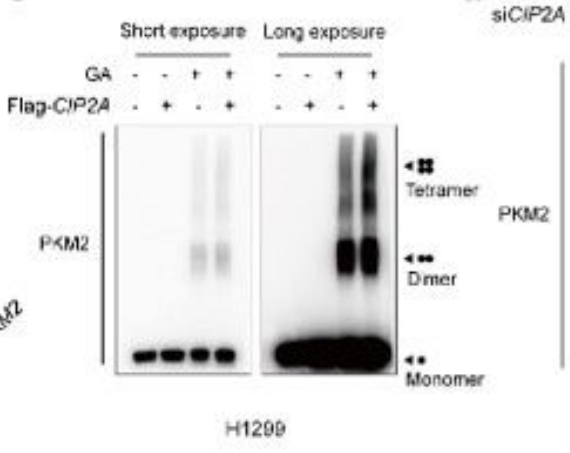

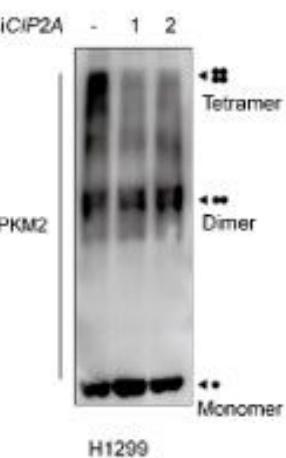

H1299

b
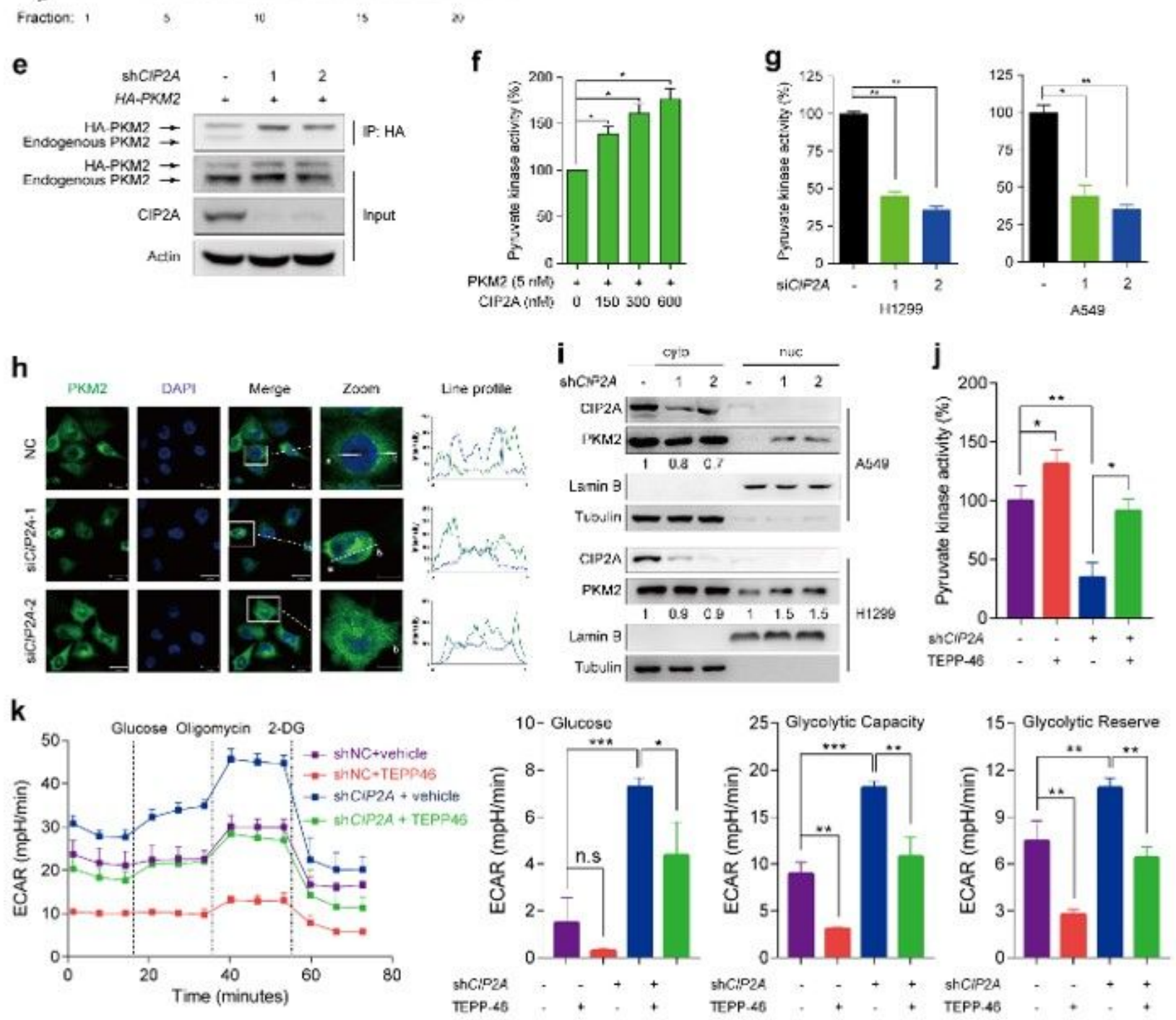

Fig. 3

Figure 3

CIP2A inhibits glycolysis by stabilizing oligomerization, upregulating the activity and hindering the nuclear translocation of PKM2. (a) CIP2A knockdown shifts PKM2 to lower molecular weight fractions. Cell lysates from $A 549$ cells that were transfected with sh CIP $2 A$ were separated by size-exclusion chromatography and analyzed by western blotting. The fraction number and relative molecular weight (Mr) are indicated. (b) CIP2A shifts PKM2 to higher molecular weight fractions. The indicated plasmids 
were cotransfected into 293T cells, and cell lysates were separated by gel filtration, followed by western blotting analysis. The fraction number and Mr are indicated. (c) Cell lysates from H1299 cells transiently expressing Flag-CIP2A were incubated with or without the crosslinking reagent $0.01 \%$ glutaraldehyde and then subjected to western blot analysis using an anti-PKM2 antibody. GA, glutaraldehyde. (d) H1299 cells were transfected with siCIP2A for $36 \mathrm{~h}$, lysed, and the cell lysates were crosslinked with $0.01 \%$ glutaraldehyde at $37^{\circ} \mathrm{C}$ for 9 min. Immunoblot analyses were performed with an anti-PKM2 antibody. (e) Silencing of CIP2A reduces the intermolecular interactions with PKM2. CIP2A-knockdown H1299 cells were transfected with HA-PKM2, lysed, and subjected to coimmunoprecipitation assays to examine the interactions of endogenous PKM2 with HA-PKM2. IP, immunoprecipitation. (f) Pyruvate kinase activity of bacteria-expressed PKM2 was measured in bacteria expressing different concentrations of purified CIP2A protein. The relative activity of PKM2 was set to $100 \%$. Data were compared among groups using unpaired Student's $t$ test. * $P<0.05$. (g) H1299 and A549 cells were transfected with siNC, siCIP2A-1 or siCIP2A-2 for 48 hours later, lysed, and the lysates were assayed for pyruvate kinase activity. One-way ANOVA, ${ }^{*} P<0.05,{ }^{*} P<0.01$. (h) Subcellular localization of PKM2 in $\mathrm{H} 1299$ cells transfected with siCIP2A was examined by immunofluorescence assay. Cells were immunostained with an anti-PKM2 antibody (green), DAPI (blue) was used to mark the nucleus. The line profiles of PKM2 and DAPI signals were determined with ZEN 2011 (Carl Zeiss) software. (Scale bars, $30 \mu \mathrm{m}$ for unmagnified image and 10 $\mu \mathrm{m}$ for magnified image). (i) Nuclear and cytosolic lysates derived from A549 and H1299 cells transfected with sh CIP2A were separated, and total PKM2 was determined by western blot. Nuclear lamin $B$ and cytoplasmic tubulin were used as controls. Cyto, cytoplasm; Nuc, nucleus. (j, k) H1299 cells stably expressing CIP2A shRNA were treated with vehicle (DMSO) or $30 \mu \mathrm{M}$ TEPP-46 for 36 hours and subsequently subjected to pyruvate kinase activity $(\mathbf{j})$ and ECAR $(\mathbf{k})$ analysis. Data represent the average of three independent experiments (mean \pm SD). ${ }^{\star} P<0.05,{ }^{*} P<0.01$, ${ }^{\star \star \star} P<0.001$; n.s., not significant for the indicated comparison. 
a
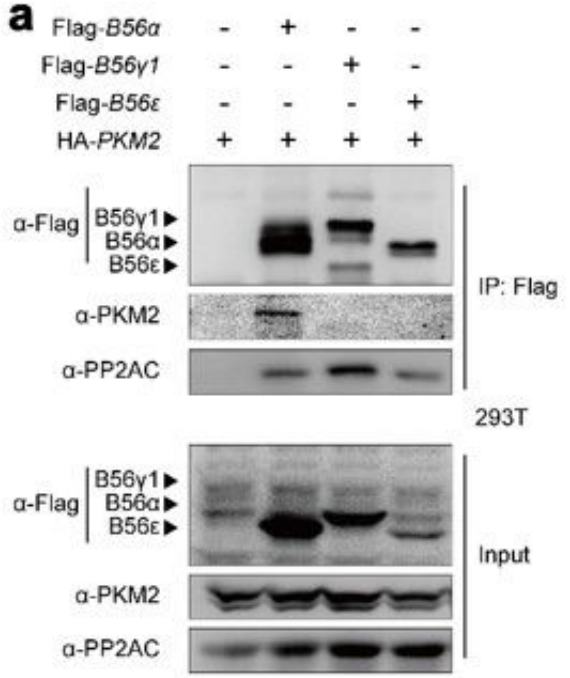

f

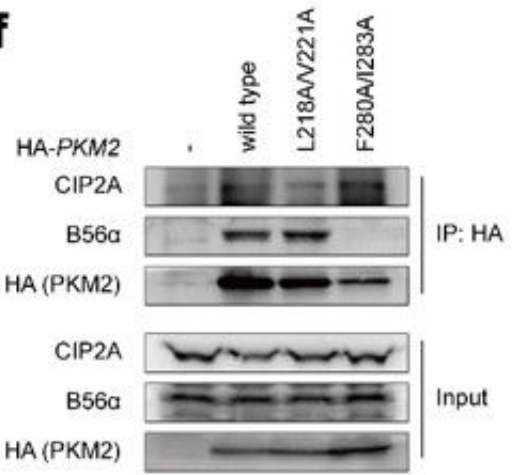

b
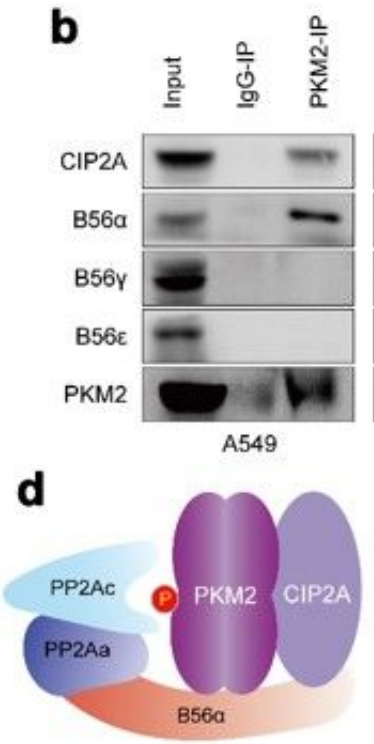

g

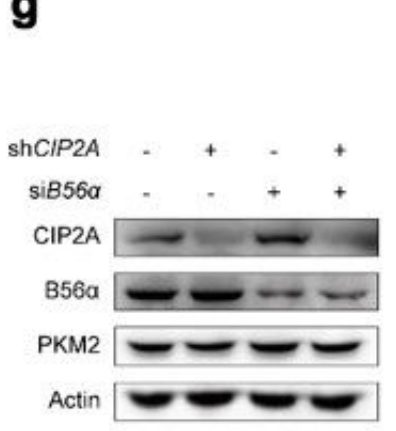

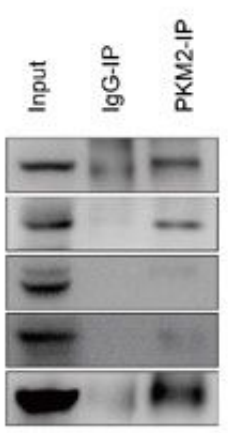

C

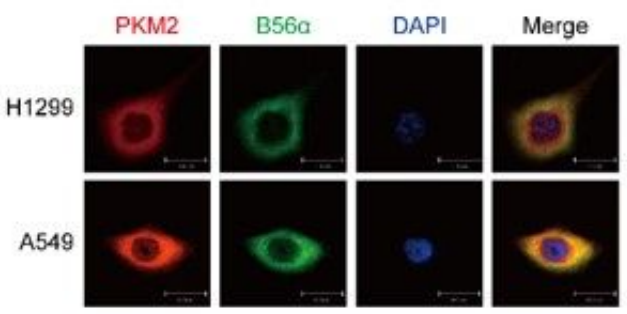

H1299

e

$\begin{array}{lll}\mathbf{S} 222 & \mathrm{~S} 243 & \mathbf{S} 249\end{array}$ 216- VDLPAV SEKDIQDLKFGVEQDVDMVFASFIRKASD -250 \begin{tabular}{l|l|l|l|l|l|}
\hline PKM & N & A1 & B & A2 & C \\
\hline
\end{tabular}

278- RRFDEIILEASDGIMVARGDLGIEIPAEKVFLA -309 $\mathrm{S} 287$
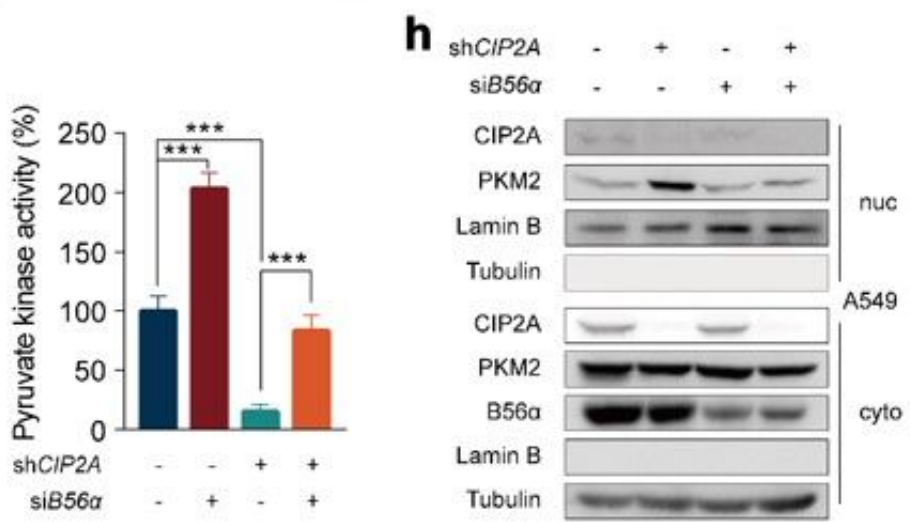
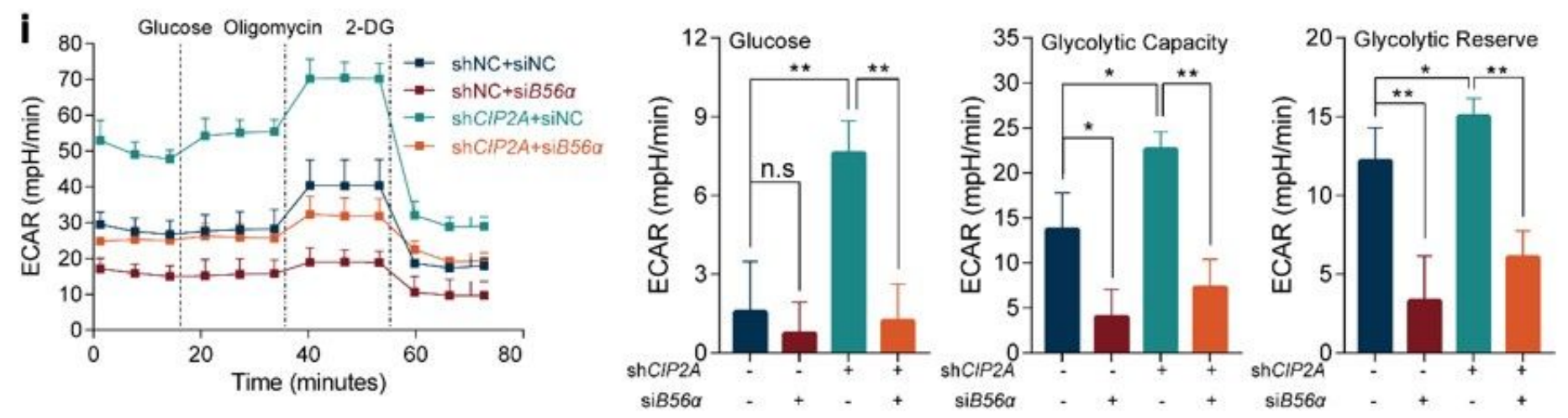

Fig. 4

\section{Figure 4}

B56a regulates PKM2 activity and metabolism. (a) Indicated constructs were cotransfected into 293T cells, and total protein extracts were immunoprecipitated and analyzed by western blot. (b) Endogenous PKM2 was immunoprecipitated from A549 and H1299 cell lysates and subsequently analyzed by western blotting using the indicated antibodies. (c) Immunofluorescence assays of H1299 and A549 cells using antibodies against PKM2 (red) and B56a (green), and DAPI to stain nuclei (blue). Scale bars, $30 \mu \mathrm{m}$. (d) Schematic representation of the ternary complex composed of the PP2A complex, PKM2 and CIP2A. (e) Schematic representation of the protein domains of PKM2 and the location of two potential B56a binding 
motifs and surrounding serine that might be modified. (f) Indicated HA-PKM2 mutant plasmids were transfected into $\mathrm{H} 1299$ cells, and proteins immunoprecipitated by HA-PKM2 were analyzed by western blotting. (g-i) A549 cells stably expressing CIP2A shRNA were transfected with siRNA targeting B56a or control for 36 hours, and then pyruvate kinase activity (g) and subcellular localization of PKM2 (h) and ECAR (i) were determined. One-way ANOVA, $* P<0.05$, ** $P<0.01$, $* \star * P<0.001$. Cyto, cytoplasm; Nuc, nucleus.
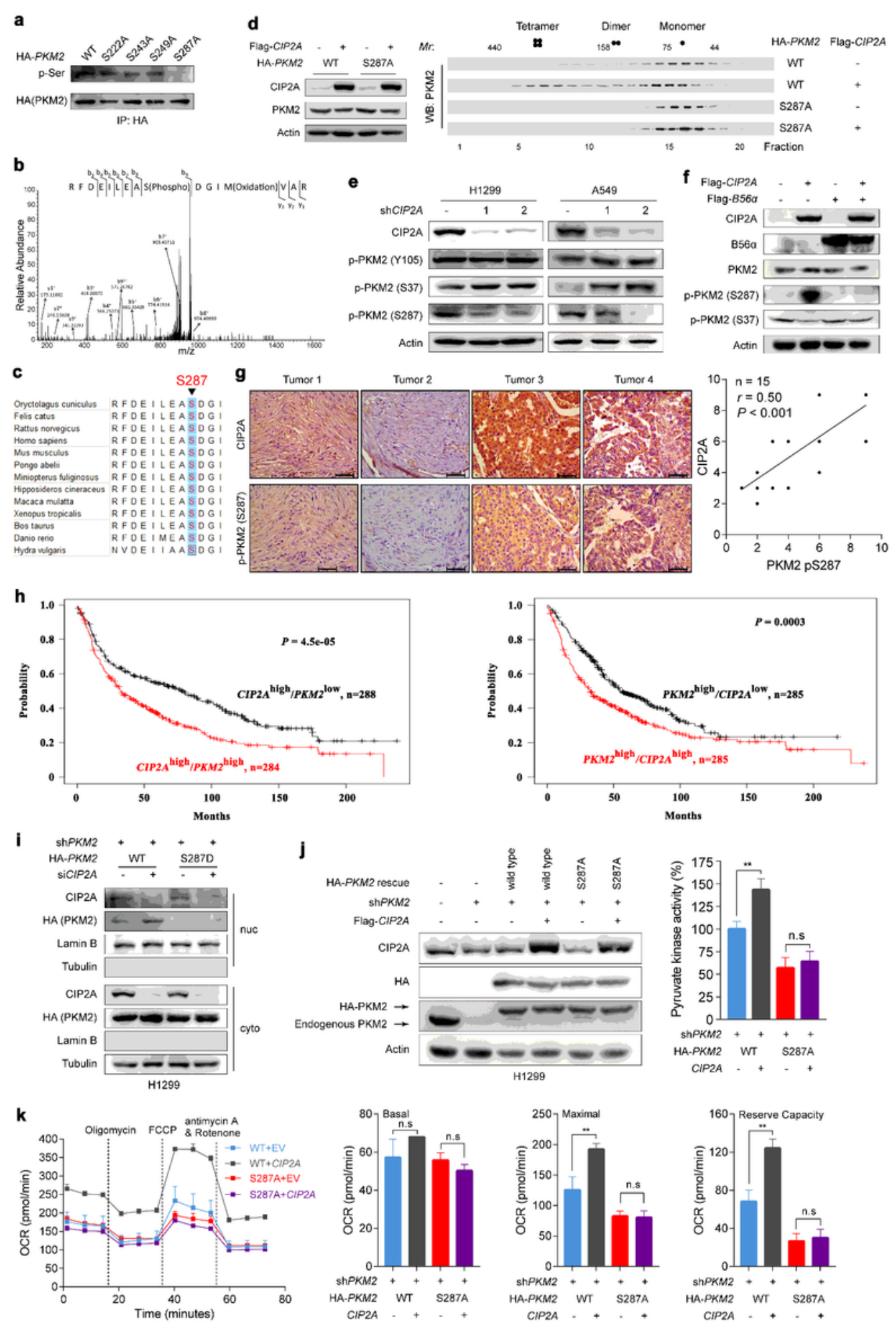

Fig. 5

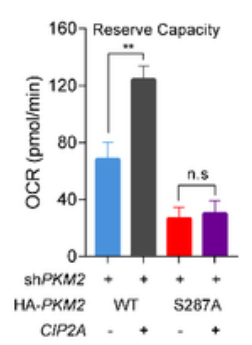




\section{Figure 5}

S287 is a critical phosphorylation site of PKM2. (a) S287A decreases PKM2 phosphorylation. The indicated HA-PKM2 mutant plasmids were transfected into 293T cells, and protein was immunoprecipitated for phosphorylation analysis. IP, immunoprecipitation. (b) Mass spectrometry shows PKM2 S287 phosphorylation. (c) S287 of PKM2 is evolutionarily conserved in the indicated species. The sequences around PKM2 S287 from different species were aligned. (d) CIP2A shifts PKM2 wild type but not S287A mutant PKM2 to higher molecular weight fractions. The indicated plasmids were cotransfected into 293T cells, and cell lysates were separated by size-exclusion chromatography, followed by western blot analysis. Fraction numbers and relative molecular weights ( $\mathrm{Mr}$ ) are indicated. Ectopic expression of Flag-CIP2A and HA-tagged wild type PKM2 or S287 PKM2 mutants in 293T cells was detected by western blot. (e) Western blot analyses of PKM2 phosphorylation at Y105, S37 and S287 in A549 and H1299 cells transfected with of sh CIP2A. (f) western blot analysis of PKM2 phosphorylation at S287 and S37 in H1299 cells that ectopically coexpressed Flag-CIP2A and Flag-B56a. (g) Immunohistochemical staining experiments were performed in 15 human lung adenocarcinoma specimens using anti-CIP2A and anti-phospho-PKM2 Ser287 antibodies, and the correlation of the immunoreactivity score between CIP2A and PKM2 S287 phosphorylation was analyzed. Note that some scores overlap, and the dots on the graphs indicate more than one sample. Scale bars, $50 \mu \mathrm{m}$. (h) Overall survival of NSCLCs with high or low expression levels of CIP2A and PKM2. Data were obtained from the Online Survival Analysis Software ${ }^{43}$ (http://kmplot.com/analysis/index.php? $\mathrm{p}=$ service\&cancer=lung). (i) Cytosolic and nuclear fractions were prepared from $\mathrm{H} 1299$ cells transfected with shPKM2, and shRNAresistant HA-PKM2 (wild type or S287D), and siCIP2A. Nuclear lamin B and cytoplasmic tubulin were used as controls. Cyto, cytoplasm; Nuc, nucleus. (j-k) Flag-CIP2A and reconstituted shRNA-resistant HAPKM2 (wild type or S287A mutant) were transfected into shPKM2-treated H1299 cells. Pyruvate kinase activity $(\mathbf{j})$ and OCR mitochondrial respiration parameters $(\mathbf{k})$ were measured. PKM2 silencing and reexpression efficiency were verified by western blot. Data represent the average of three independent experiments (mean $\pm \mathrm{SD}$ ). EV, empty vector. ${ }^{*} P<0.05, * * P<0.01 ;$ n.s., not significant for the indicated comparison. 


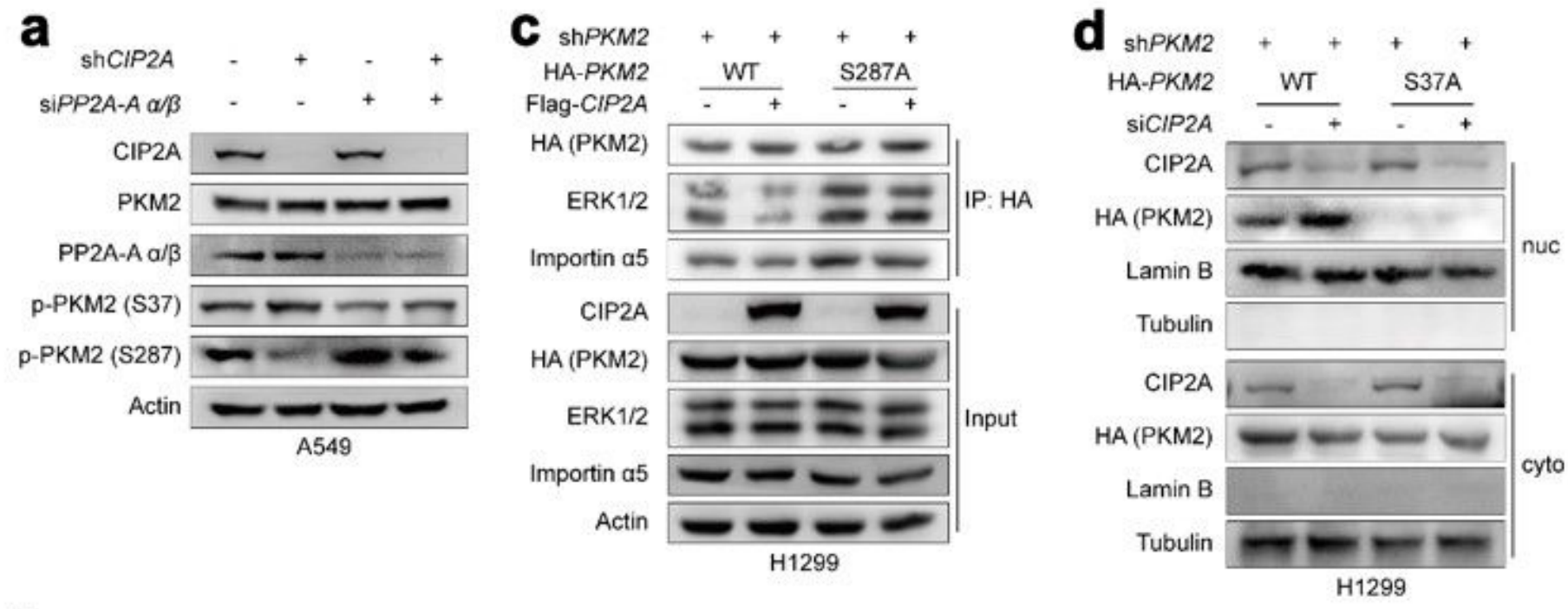

b

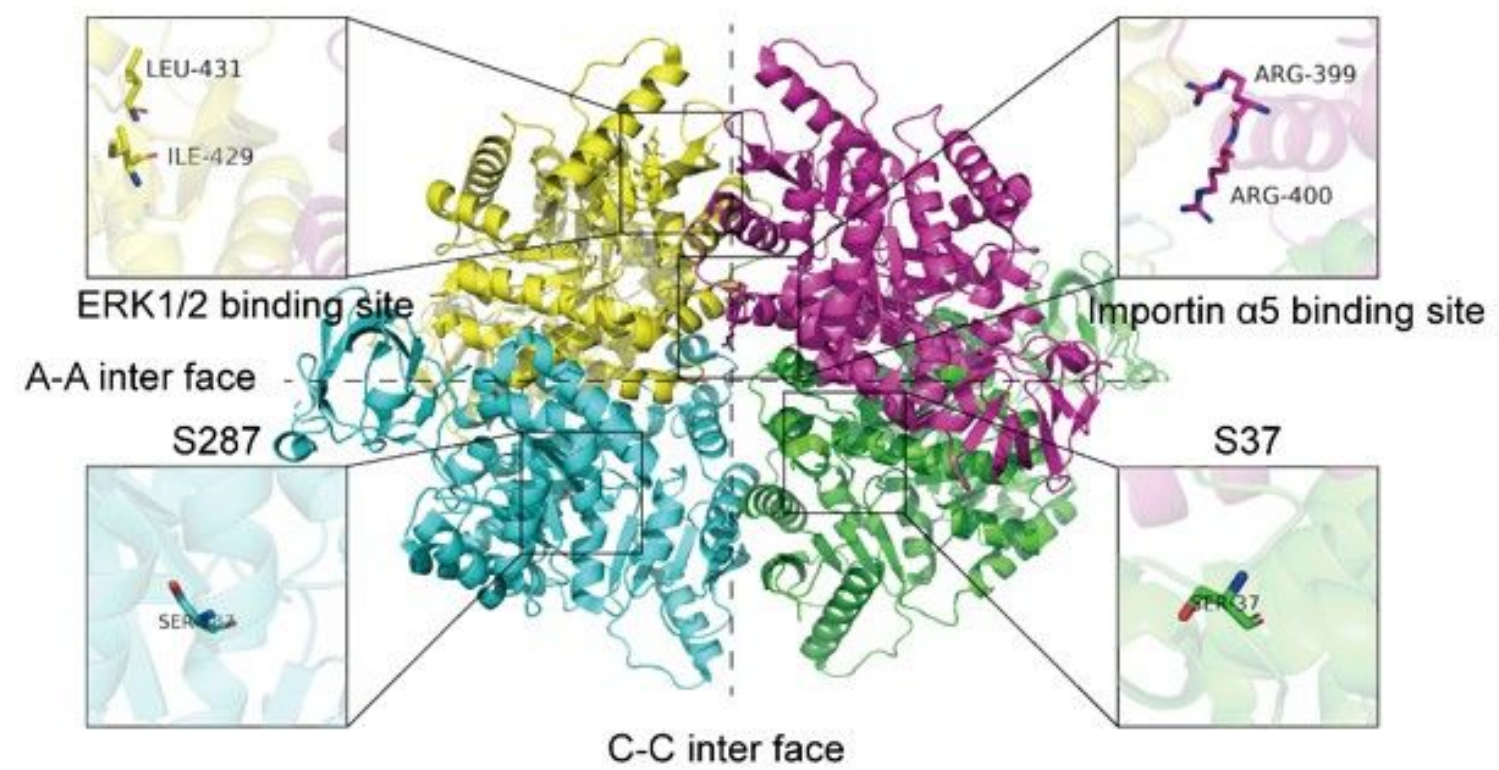

Fig. 6

Figure 6

S287 affects PKM2 binding to ERK1/2 and importin a5. (a) Knocking down PP2A blocks CIP2A depletioninduced S37 phosphorylation. A549 cells stably expressing CIP2A shRNA were transfected with PP2A-A $a / \beta$ siRNA or control, followed by immunoblotting analysis. (b) Structural analysis of the human PKM2 tetramer (PDB ID code 3SRD). The four PKM2 monomers are shown in cartoon mode with different colors, and the interfaces between two dimers are represented by dashed lines. The amino acid residues Ile 429/Leu 431 (ERK1/2 binding sites), Arg399/400 (importin a5 binding site), Ser 287 and Ser 37 are indicated by ball and stick models and colored yellow, purple, cyan and green, respectively. Magnified images with labelled residues are also presented. (c) Association among PKM2, ERK1/2 and importin a5. Flag-CIP2A and shRNA-resistant HA-tagged PKM2 (wild type or S287A mutant) were transfected into 
PKM2-depleted H1299 cells, followed by immunoprecipitation and western blotting analysis with the indicated antibodies. (d) Cytosolic and nuclear fractions were prepared from $\mathrm{H} 1299$-sh PKM2 cells transfected with shRNA-resistant HA-PKM2 (wild type or S37A mutant) and siCIP2A. Nuclear lamin B and cytoplasmic tubulin were used as controls.

a

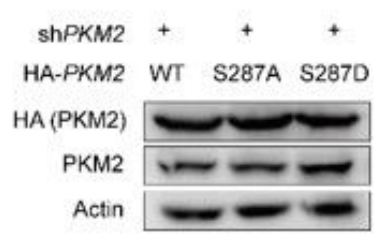

d

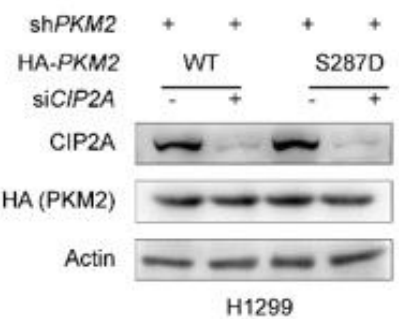

e

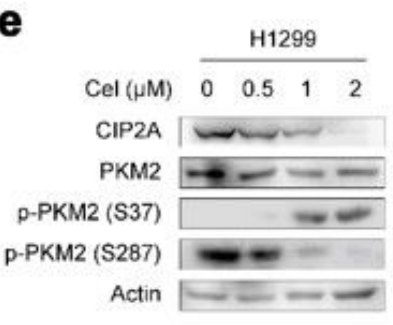

g

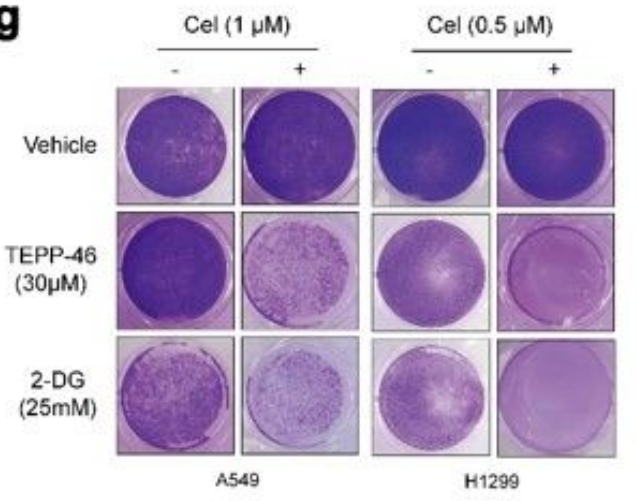

b

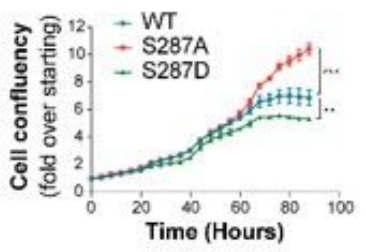

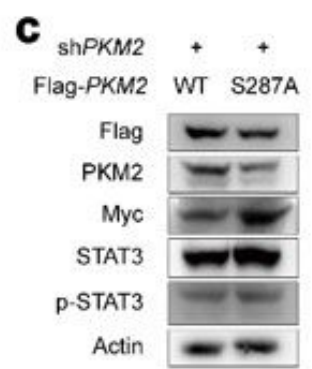
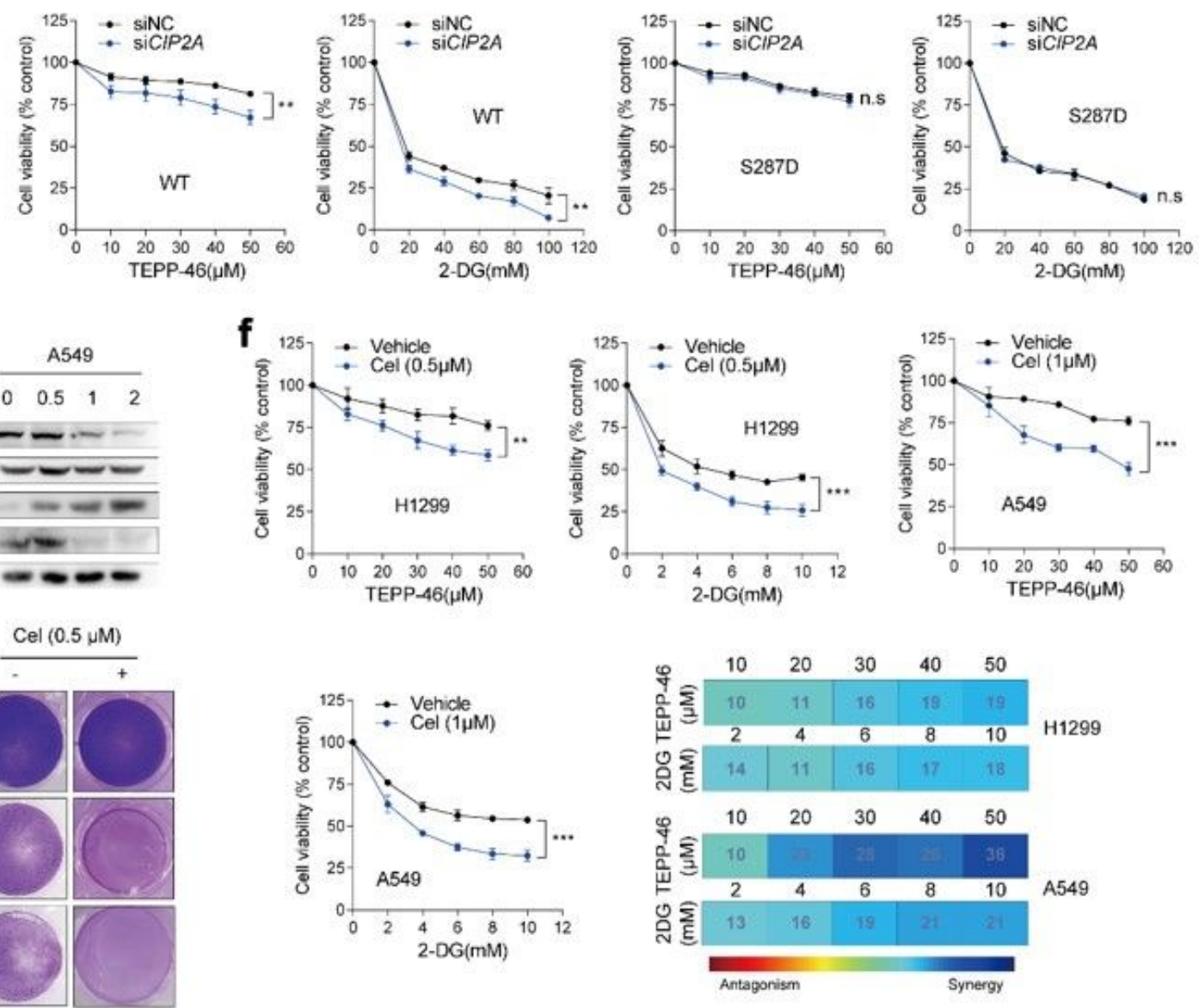

Fig. 7

Figure 7

Modulation of CIP2A and PKM2 controls NSCLC cell proliferation. (a) H1299 cells were transfected with shPKM2 and shRNA-resistant PKM2 (wild type or mutants), and cell growth was determined by the cell confluence area. Student's $t$ test. ** $P<0.01$, *** $P<0.001$. (b) H1299 cells were transfected with shPKM2 and shRNA-resistant PKM2 mutants, and images were acquired and quantified 10 days after cells were seeded in 96-well ultralow attachment plates (300 per well). Student's $t$ test, * $P<0.05$, ** $P<0.01$. (c 
Western blot analyses of indicated proteins in $\mathrm{H} 1299$ cells that were transfected with shPKM2 and shRNA-resistant Flag-PKM2. (d) H1299 cells were transfected with shPKM2, shRNA-resistant HA-PKM2 (wild type or mutant), and siCIP2A. The cells were then treated with indicated compounds for $48 \mathrm{~h}$, and cell viability was measured by CCK-8 assay. Two-way ANOVA, $* * P<0.01$; n.s., not significant. (e) H1299 and $A 549$ cells were treated with celastrol for $48 \mathrm{~h}$, lysed, and cell lysates were analyzed by immunoblotting. (f) Cell viability of A549 cells treated with indicated compounds (upper and left panels), and the combinatory effects were analyzed by Bliss synergism analysis (lower right panel). Two-way ANOVA, $* \star \star ~ P<0.01$. (g) Crystal violet staining of A549 and H1299 cells after seeding in 12-well plates and treatment with the indicated compounds for $48 \mathrm{~h}$.

a

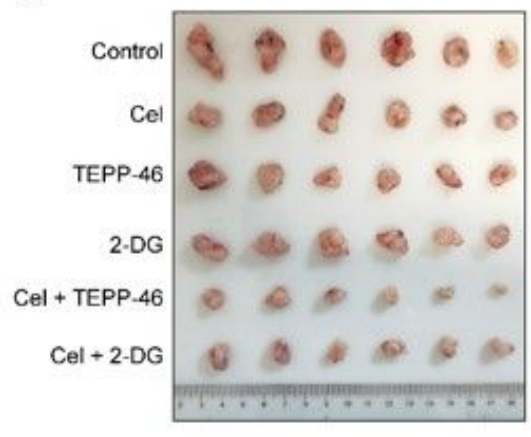

e

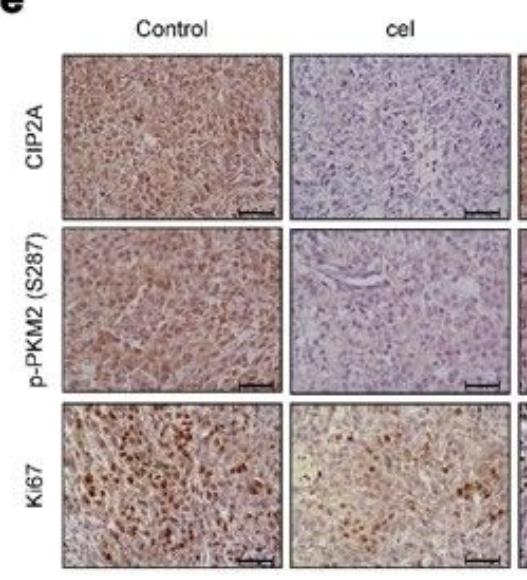

b

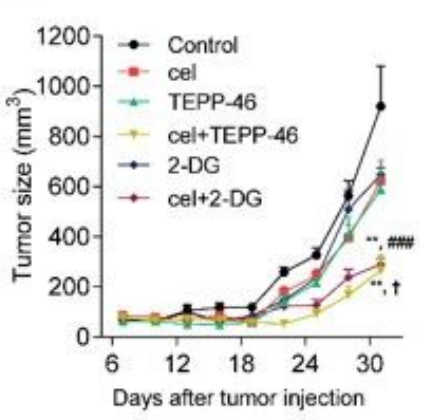

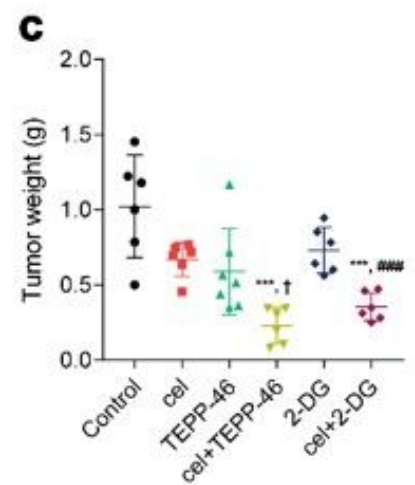

\section{d}
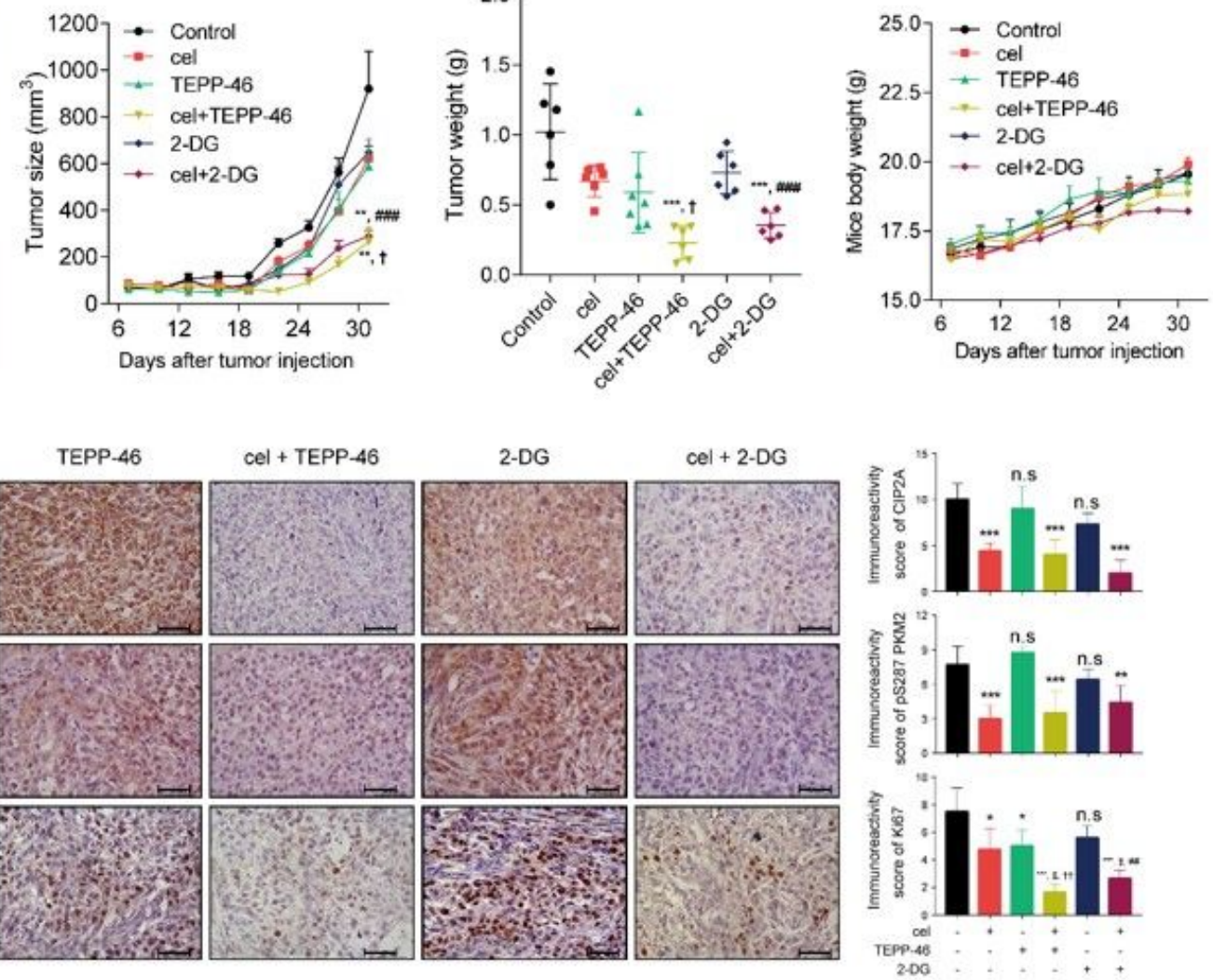

\section{f}
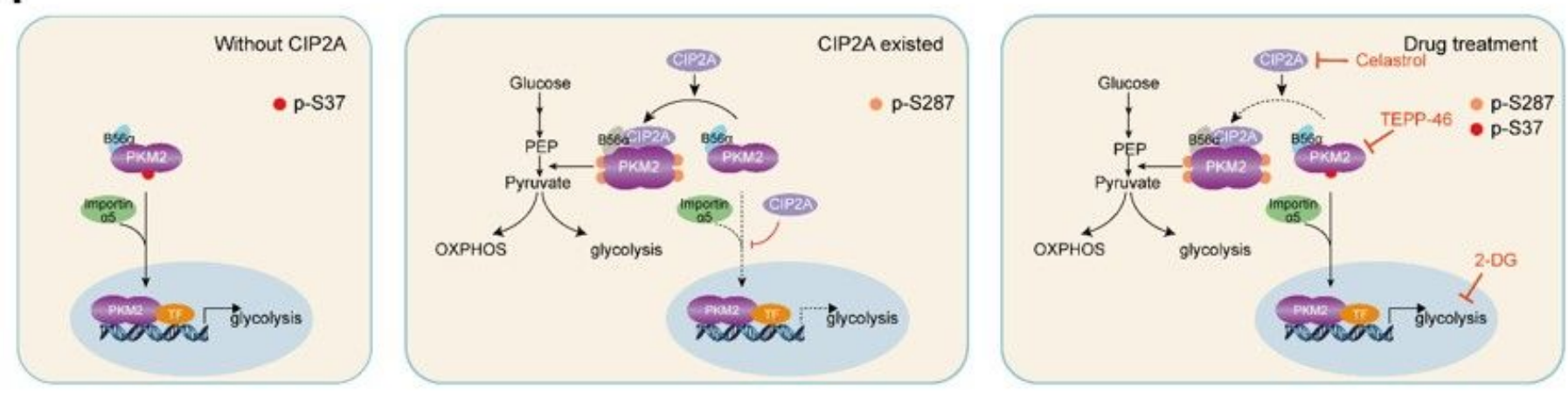

Fig. 8 
Effects of combinatory modulations of PKM2 and CIP2A on NSCLC xenograft model. (a-d) Nude mice bearing A549 cells were treated with celastrol ( $2 \mathrm{mg} / \mathrm{kg}$, orally daily) and/or TEPP46 ( $100 \mathrm{mg} / \mathrm{kg}$, orally daily) and/or 2-DG (500 mg/kg/day, intraperitoneal injection), and tumor volume was measured every 3 days. Ex vivo images of resected tumors (A), growth curves of the tumor volume (b), tumor weight (c) and body weight (d) are shown, respectively. ${ }^{*} P<0.01, * \star * P<0.001$ indicate combination group versus celastrol group; $† P<0.05$ indicates combination group compared to TEPP-46 group; \#\#\# indicates $P<$ 0.001 combination group versus 2-DG group (two-tailed Student's $t$ test). (e) Representative immunohistochemistry staining image for CIP2A, phospho-PKM2 at S287 and Ki67 expression in resected xenograft tumours (left panel). The immunoreactivity scores were quantified (right panel). Scale

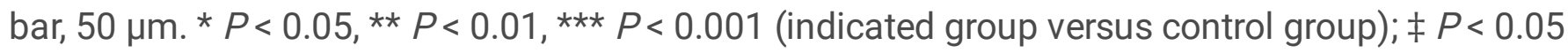
(combination group vs celastrol group); †† $P<0.01$ (combination group vs TEPP-46 group); \#\# $P<0.01$ (combination group vs 2-DG group; two-tailed Student's $t$ test). (f) Proposed model summary of CIP2A modulation of metabolic pathways by regulating PKM2 phosphorylation.

\section{Supplementary Files}

This is a list of supplementary files associated with this preprint. Click to download.

- SupplementaryMaterials.docx 